\title{
sciendo
}

BULGARIAN ACADEMY OF SCIENCES

CYBERNETICS AND INFORMATION TECHNOLOGIES • Volume 21, No 2

Sofia $2021 \quad$ Print ISSN: 1311-9702; Online ISSN: 1314-4081

DOI: $10.2478 /$ cait-2021-0024

\section{Role of Clustering, Routing Protocols, MAC protocols and Load Balancing in Wireless Sensor Networks: An Energy-Efficiency Perspective}

\author{
Nagarajan Munusamy ${ }^{1}$, Sneha Vijayan ${ }^{1}$, Ezhilarasi $M^{2}$ \\ ${ }^{1}$ KSG College of Arts and Science, Coimbatore, India \\ ${ }^{2}$ Sri Ramakrishna Engineering College, Coimbatore, India \\ E-mails: mnaagarajan@gmail.com_snehamaelin05@gmail.com mezhilarasi@gmail.com
}

\begin{abstract}
Wireless networks play an important role in science, including medicine, agriculture, the military, geography, and so on. The main issue with a network of wireless sensors is how to manage resource utilization to extend its lifetime. This paper investigates the various aspects of increased energy usage that may improve network life. Variables related to energy consumption and various performance metrics are investigated in terms of energy efficiency. To investigate how the network's energy usage can be managed, a quick overview of clustering protocols, routing protocols, MAC protocols, and load balancing protocols is conducted. This paper can provide researchers with an idea of the various parameters that influence energy consumption and what methodologies could be adapted by each parameter to conserve energy, thereby extending the network's lifetime.
\end{abstract}

Keywords: Energy, clustering, routing, MAC, duty cycle, load balancing.

\section{Introduction}

A Wireless Sensor Network (WSN) is a collection of sensor nodes that are set up to map a geographical area with physical phenomena. All of WSN's applications are applied to trends in animal monitoring, climate management, medical applications, military control, and facility maintenance. The challenge for WSN is to extend its life because each sensor has a small battery capacity and it needs to feel, transmit, retrieve, and process with this limited capacity.

According to Ezh il ar a s i and Kris h nave n i [1], a certain amount of power is consumed at each stage, resulting in a decrease in battery capacity. The energylimited source is critical for the wireless sensor network. Different nodes in the WSN record values, which are continuously transmitted to the base station via node-tonode communication and cluster heads. The data is collected, aggregated, and sent to the base station by the cluster heads. The sensor node's energy level is primarily focused on the WSN battery. 


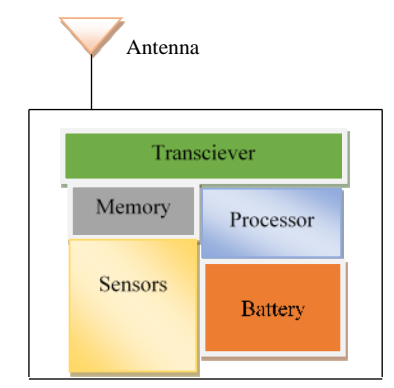

Fig. 1. Block-diagram of a WSN system

$\mathrm{Nagarajan}$ and Karthikeyan [2] indicate that it is difficult to manage the battery multiple times when looking at remote locations where sensors must be deployed using small aircraft. Routing is always difficult in such a scenario if nodes are lost due to excessive energy consumption. Data collected in sensor nodes generate a large amount of network traffic during transmission, which can be mitigated by an effective composite detection mechanism. According to $\mathrm{Hay}$ a a A adi et al. [3] efficient power management is required to improve network lifetime due to limited source capacity. As a result, resource utilization should be optimized in order to extend network life. Tife $\mathrm{n}$ R $\mathrm{Ra}$ lt, B ou abdallah and Challah [4] explain that WSN operates in three modes: communication, sleep, and estimation. During communication, the nodes expend a significant amount of energy, which is directly proportional to the distance capacity. To increase the lifespan of a WSN, we must focus more on the communicating nodes. This paper considers methodologies that can help increase the energy and life of wireless sensor networks, as well as the challenges and factors influencing network energy use. In WSN, a detailed discussion is conducted based on MAC protocols, clustering protocols, and load balancing. This paper concludes by providing the researchers with an in-depth idea to help them envision their ideas for developing an energy-efficient WSN application.

\section{Challenging issues in WSN}

The general issues in a WSN that need to be given attention are listed below by Ezhilarasi and Krishnaveni [1].

1. Environment. WSN is commonly used in situations where human intervention is unlikely. The nodes are frequently left unattended. Further node failures will occur if they are left unattended.

2. Hardware. A sensor node's four main components are a sensor unit, a processing unit, a transceiver unit, and a power unit. They must all fit into a small case that is also light. It must be able to operate on the network without human intervention, which necessitates adaptation to its surroundings.

3. Scalability. A WSN is typically application-specific. As the number of nodes increases, networks must be sufficiently scalable to conform to the situation.

4. Fault tolerance. Network-mounted nodes must be resistant to frequent faults and able to withstand even extreme environmental conditions. 
5. Deployment. Sensor nodes must be positioned in a way that is appropriate for the application, either deterministically or randomly. In the absence of careful implementation, resource consumption can be enormous, and the network can be disrupted.

6. Coverage. Sensor nodes deployed in every nook and cranny of the network must be able to collect data from all nodes.

7. Connectivity. In order to facilitate data transfer, the deployed nodes must be interconnected. If the connectivity is not working properly, there is a higher chance of node errors.

8. Energy. Because the energy nodes are heavily reliant on batteries, the primary disadvantage of a WSN is the inability to use efficiently power.

9. Data aggregation. The aggregation of data from various sensor nodes consumes more resources. Furthermore, data packet replication should be avoided because it can result in excessive energy waste.

10. Transmission. Network nodes should interact in a variety of environmental conditions such as rain, cloud, snow, and so on, using devices such as phones, optical media, infrared, etc.

The issues mentioned above are quite common in a WSN. Looking in depth at the challenging issues of WSN, Anitha and Suresh [5] categorize them as design, architecture, data aggregation, as well as the challenges involved in the network and transport layer of the network. When considering the design challenges, the first thing to consider is the network's energy efficiency. As we all know, a WSN operates on the power supplied by batteries, when the batteries run out, the network's stability suffers greatly. When considering the protocols we use in the network for communication, we must focus on developing simple protocols while making them complex to implement, which eventually adds overhead to the system's complexity. The size of a WSN is also a challenge because increasing the network's size automatically degrades the network's performance. When we deploy sensors, we must understand how our deployment strategies will affect network performance. According to Vivek Kumar Singh, R. Kumar and Sahana [6], the environment in which we deploy is also a major concern because the sensors must be adaptable to the environment in which we deploy, which means they must be able to sustain themselves even when deployed in harsh conditions. The WSN faces numerous real-time challenges, such as data delivery and response times that must be fast enough to allow for necessary action. When considering applications such as forest fire detection and weather monitoring, an immediate response is required with no delay. In such cases, the routing protocols used in the network must be as fast as possible. When we look at the architecture of a WSN, we must consider routing, hardware, and implementation. The software serves as a link between the network hardware and the system utilities, and data transmission, monitoring, and encoding must all take place concurrently. The progress of communication is heavily reliant on data sensing, calculation, and processing. When the network topology changes, it is necessary to update it across the network while using the least amount of energy for transmitting messages about the topology change. The network must be adaptable to a wide range of protocols and applications, and transmission accuracy must be 
controlled. When collecting data, we must be mindful of the energy consumed while increasing the speed of the data path and the rate of radio transmission, because network performance is determined by processing speed and communication rate. In the case of data aggregation, the unreliability of a sensor network poses a significant challenge. Wang [7] discusses how the lack of required data or incomplete information from deployed sensors makes data aggregation a stressful process. Protocols must be designed to eliminate redundancy while also focusing on reducing power consumption. It is suggested that improved clustering techniques be used for this purpose.

When it comes to the network layer of a WSN, Anitha and Suresh [5] discuss the challenges of power efficiency, multipath, platform, design, and maintenance. To make the best use of power in a WSN, an efficient route must be discovered prior to transmitting data among network nodes, as route selection can determine the network's lifespan. The routing protocol proposed for the network must be capable of dealing with multipath designs. To perform path discovery, a flexible and adaptable platform is required, and the system design must overcome redundancy while also making good use of bandwidth and improving energy efficiency. End-toend reliable connections are required in the transport layer to split packets at the transmitter and rejoin them at the receiver. The most important requirement of a transport protocol is that segments be transmitted in an orderly fashion in order to ensure reliability. The transport layer is responsible for delivering packets to multiple sensors at the same time regardless of the condition. When the network's bandwidth is low, there is a greater chance of congestion, which results in packet loss. Some external factors, as well as the predetermined deployment of sensor nodes, can have an impact on WSN communication. All of these issues must be addressed when designing an effective WSN for applications.

\section{Clustering and routing protocols}

To save energy in WSN, sensor nodes are clustered based on a set of criteria and data is gathered at each Cluster Head $(\mathrm{CH})$ to avoid redundant data transfer to the sink. Furthermore, the collected data is efficiently routed to the sink via an intelligent routing process. In this section, we will look at a few routing protocols that help with effective clustering and routing in WSNs.

Jiang, Yuan and Zhao [8] use FLOC (Fast LOcal Clustering), which creates clusters of similar size with nodes that do not overlap during cluster creation. When we consider protocols that can encourage low energy consumption, reduce transmission costs, and balance the load, Rathna [9] examine that we need to perform a broad review to categorize the protocols according to our requirements. Xuxun Liu [10] use DWEHC (Distributed Weight-based protocol for Energyefficient Hierarchical Clustering), which generates balanced clusters with no overlaps and distributes them in the network using the Weight dependent Protocol. To some extent, these two algorithms appear to be the most effective at conserving energy in WSN. According to $\mathrm{Ch}$ u n y a o Fu et al. [11], the TDMA-based MAC protocol, an energy-aware protocol implemented in most WSNs, is Low Energy Adaptive 
Clustering Hierarchy (LEACH). This protocol's primary goal is to reduce energy consumption and set up data transfer clusters for base stations. The stochastic algorithm is used to generate sensor nodes after each round, and the CH's function is to accumulate, compress, and transmit data. It relays data from the cluster to the base station using resident data calculation. This protocol is best suited for uniform networks and not for heterogeneous networks. LEACH, the most efficient energy conservation-based algorithm in WSN, employs a one-hop topology with random cluster head selection. The cluster heads are unstable and dispersed at random. The energy dissipation algorithm has not been balanced, despite the fact that it has been tailored to various WSN applications. LEACH-C outperforms LEACH based on the same metrics, except that it suggests cluster formation, as proposed by $\mathrm{Tr}$ i p a th $\mathrm{i}$ et al. [12]. The LEACH-C protocol is based on clusters, which select the $\mathrm{CHs}$ from the base station. The amount of energy in the nodes is transmitted to the base station at the start of each round. The $\mathrm{CHs}$ are chosen by the base stations based on the measurements sent by the nodes. When the sensor nodes are close to the base station, this protocol does not produce better results than the LEACH method. The main disadvantage of this protocol is that the $\mathrm{CHs}$ are not distributed. The cluster was formed using the residual energy of the nodes. B o T a ng, W ang and Z h an $\mathrm{g}$ [13] use GEAR (Geographic and Energy Conscious Routing) which claims to achieve energy efficiency by taking into account a variety of factors such as source and destination location, network density for interrupted contact, and energy costs. S qatish Chand, Singh and Kumar [14], focus on Hybrid Energy Efficient Distributed (HEED), an efficient, energy-efficient algorithm that takes neighbouring nodes into account during cluster head distribution and randomly selects the cluster head, which changes during each iteration. To increase network lifetime, the HEED protocol is proposed. CHs are chosen on the basis of residual energy and node density. Han et al. [15] use the spanning tree technique to focus on energy efficiency. The GSTEB algorithm demonstrates efficient routing by constructing spanning trees, which has resulted in an increase in network lifetime. Is hu Sharma, Singh and Khurana [16] focus on PEGASIS (Power-Efficient Selection in Sensor Information Systems) as an optimal chain-based protocol aimed primarily at improving energy efficiency. A research study reports that collecting input from sensor nodes based on fixed settings improves energy efficiency by $50 \%$, but it creates data overhead. Power Efficient Gathering in Sensor Information Systems (PEGASIS) is a hierarchical protocol that uses a greedy algorithm to transfer packets to the base station in a sequential order. To relay data, the nodes form a chain, and if any node in the chain fails, the chain must be rebuilt. Each sensor node within the deployment area serves as the $\mathrm{CH}$ for sending data to the basic station. The Deterministic Energy Efficient Clustering (DEC) protocol, according to A $1 \mathrm{~m}$ a s $\mathrm{Z}$ a r g h a m [17], promises a better option for selecting CHs. Communication can be completed with a single hop or multiple hops. Only single data packets are sent to neighbours in a single hop, whereas multi-hop data packets are sent simultaneously to more neighbours, consuming more resources. B o m g n i et al. [18] propose the Improved Permutation-based Routing Protocol (IPRP) for high energy conservation. It resolves the debate over infinite memory capacity, which is present in the majority 
of routing protocols. S a bet and Naji [19] propose Multiple Level Route aware Clustering (MLRC) to distribute energy among sensor nodes via route consciousness. It keeps the drain rate constant based on the amount of data collected, stored, and transmitted over the network. Y a s h a Is t w a l and V e r m a [20] employ the Dual Cluster Head Routing Protocol (DCHRP), which focuses on maximizing network lifetime through three levels of heterogeneity in $\mathrm{CH}$ selection. The ultimate goal of this protocol is to limit $\mathrm{CH}$ selection in order to reduce energy consumption, as more energy is required during $\mathrm{CH}$ selection. This protocol reduces the number of clusters, and the three levels of heterogeneity are achieved through three stages: $\mathrm{CH}$ selection, cluster formation, and finally communication with the base station. Delivery time, energy consumption, and data reliability are all considered. S a m a y ve e r S ing h and Malik [21] claim that the Stable Election Protocol (SEP) is a heterogeneous clustered protocol based on the weights chosen by each node, the node with the most remaining energy becomes the $\mathrm{CH}$. It has two types of nodes: regular nodes and specialized nodes. Advanced nodes require more resources than normal nodes for data aggregation and transfer. K a i P e $\mathrm{ng}$ [22], propose the use of PEDAP (Power Efficient Data Collection and Aggregation Protocol), which is a framework of two algorithms that, using optimal spanning trees, focuses on power efficiency as its primary goal. Han and $\mathrm{Zhang}$ [23] propose the Weighted and Parameter Optimization-based Energy-Efficient Clustering Routing Protocol (WPO-EECRP) to improve energy efficiency by reducing the number of neighbours, hops, and internode distances between base stations and sensor nodes in the network. This protocol is said to extend the network's lifespan. Mazumdar and Om [24] Distributed Unequal Cluster-based Routing (DUCR) address the hotspot problem by employing unequal clusters, the size of which decreases as they approach the sink. The node with the most energy is chosen as the $\mathrm{CH}$, and an energy-efficient routing algorithm is used to balance load among cluster heads. Saranya, Shankar and $\mathrm{K}$ a $\mathrm{n}$ a g a c hi d a m b are s an [25] describe the EECS (Energy Efficient Clustering Scheme), which employs balanced clustering to select cluster heads and incorporates various parameters to achieve energy efficiency. Darabkh's et al. [26] Efficient and Energy-Aware Clustering and Routing Protocol (EA-CRP) claims to reduce energy consumption among network sensor nodes. The deployment field is divided into layers, with each layer composed of a number of clusters, and the depth of the layers decreasing towards the sink. The network's lifetime is divided into rounds, with the sink node determining the time for each round. There are double $\mathrm{CHs}$ for energy conservation, with one acting as a Leader Head and the other as a Cluster Head. The Leader Head collects and aggregates data from cluster members. The Cluster Head's role is simply to relay data between layers until it reaches the sink. The Leader Head is chosen based on the node's energy, and the Cluster Head is chosen based on the node's energy as well as its distance from the sink. This protocol's major flaw is that it generates an excessive number of control messages, which reduces network lifetime. $\mathrm{Zh} \mathrm{ou}$ and $\mathrm{Yu}$ [27] propose the Balanced Energy Efficient Routing Protocol (BEERP), which is concerned with maintaining the drain rate based on the network's inner and outer regions. It aggregates data using the uniformity principle and uses the shortest paths for effective routing. $\mathrm{Zh}$ ang, M. 
Li u and Q. Li u [28] Improved Clustering by Fast Search and Finding of Density Peaks (ICFSFDP) balances the rate of energy consumption by taking into account the residual energy of the sensor nodes. It solves the problem of unbalanced energy, which is the cause of the energy hole. It defines a reactive threshold for deferring the death of the network's first node. $\mathrm{H}$ o s e n and Ch o [29] propose the Energy-based Cluster Centred Routing Protocol (ECCRP), which ranks cluster heads. The node's ranks are estimated based on certain parameters, and these ranks are periodically circulated among the nodes using the piggy-banking concept. During cluster formation and cluster head selection, this protocol reduces the number of control messages sent. It has been proven to increase network longevity by lowering energy consumption rates. The Integrated Ant Colony Optimization and Power Energy GAthering in Sensor Information Systems (IACO-PEGASIS) is a chain-based routing protocol proposed by $\mathrm{R} \mathrm{am} \mathrm{luckun}$ and B a s o [30] that optimizes the clustering process by reducing data duplication, minimizing distance between neighbouring nodes, and controlling data transmission delay. According to Robertus Bria, Wahab and Alaydrus [31], the Threshold responsive Energy Efficient Network (TEEN) is a protocol for energy conservation that leads to network longevity. The only disadvantage of this protocol is that data cannot be aggregated quickly because there is no communication between sensor nodes and CHs. Murat De ner [32] uses Adaptive Periodic Threshold responsive Energy Efficient Network Protocol (APTEEN), which is an improved version of TEEN that can aggregate and send data between sensor nodes but requires the support of a few protected transmission protocols. According to Piy us h $\mathrm{Ch}$ aran et al. [33], the DEAR protocol generates routing paths by utilizing leftover energy from a network node. D a r a b k h, E l-Y a brou di and El-M o u s a [34] proposed Balanced PowerAware Clustering and Routing Protocol (BPA-CRP) divides the network topology into equal-sized layers and clusters. A forwarder node resides in each layer and is capable of relaying data collected from CHs within the same layer as well as between layers. This protocol's clustering algorithm allows the cluster to operate across multiple rounds with no setup overhead. Every round, the forwarder node checks to see if its energy has dropped below a predefined threshold. If this is the case, a new setup phase begins to select forwarders from a new cluster. It chooses $\mathrm{CHs}$ using a random head selection process without specifying any criteria. A hierarchical routing protocol can help with significant cluster formation and $\mathrm{CH}$ selection in order to achieve the goals of increasing throughput while minimizing jitter and delay in low energy draining rate states Sengathir and Deva [35]. Improved Bkd-TreeInspired Energy-Efficiency Clustering-based routing protocol confirms significant cluster formation. It enables superior query and update performance while making extensive use of space independence. It achieves node stability, which contributes to an increase in network lifetime. It also evaluates energy consumption, delay, jitter, throughput, and the number of clusters formed as the number of sensor nodes increases. The Energy-aware Cluster-based Routing Protocol (ECRP) proposed by Noureddine Moussa, Hamidi-Alaoui and El Belrhti El Alaoui [36] demonstrate that cluster configuration is done only once during the network's lifetime. It chooses $\mathrm{CHs}$ based on residual energy in each round and uses multiple 
parameters to find the best solution. This protocol's unique feature is that it takes care of sensor nodes that do not have $\mathrm{CHs}$ within their communication range, allowing new nodes to be easily added. This protocol addresses fault tolerance issues as well as the periodic replacement of CHs. It enables cluster setup by taking into account network topology, which is done only once, reducing setup overhead. It is claimed to increase network flexibility and achieve energy efficiency. Sali m and Badran [37] describe COSEN (Chain Driven Sensor Network) as a chain-based algorithm focused on rapidly collecting data with minimal energy consumption.

Table 1. Pros and cons of the existing protocols

\begin{tabular}{|c|c|c|c|}
\hline Protocol & Year & Advantage & Disadvantage \\
\hline DWEHC [10] & 2012 & Generates balanced clusters & Depend on the weight \\
\hline LEACH [11] & 2013 & Energy aware & $\begin{array}{l}\text { Does not support heterogeneous } \\
\text { networks }\end{array}$ \\
\hline LEACH-C [12] & 2013 & Improved performance & $\mathrm{CHs}$ are not circulated \\
\hline GEAR [13] & 2013 & Interrupted deployment & Complexity in implementation \\
\hline HEED [14] & 2014 & $\begin{array}{l}\text { Node deployment is considered for } \\
\text { transmission }\end{array}$ & $\begin{array}{l}\text { Performance of the protocol depends } \\
\text { on the density of deployment }\end{array}$ \\
\hline GSTEB [15] & 2014 & Enhances network lifetime & Computational overhead \\
\hline PEGASIS [16] & 2015 & Fast transmission & $\begin{array}{l}\text { If a node in the node chain fails the } \\
\text { entire transmission collapses }\end{array}$ \\
\hline DEC [17] & 2016 & $\begin{array}{l}\mathrm{CH} \text { is elected considering different } \\
\text { parameters }\end{array}$ & Consumes more energy in multi-hop \\
\hline IPRP [18] & 2016 & Uniform energy distribution & Extra burden on $\mathrm{CH}$ \\
\hline MLRC [19] & 2016 & $\begin{array}{l}\text { Rotation of } \mathrm{CH} \text { for non drain of } \\
\text { energy }\end{array}$ & Low energy stability \\
\hline DCHRP [20] & 2017 & Formation of clusters is reduced & Complicated \\
\hline SEP [21] & 2017 & $\begin{array}{l}\mathrm{CH} \text { is chosen based on the energy } \\
\text { level of the nodes remaining }\end{array}$ & $\begin{array}{l}\text { Advanced nodes consume more energy } \\
\text { during transmission }\end{array}$ \\
\hline PEDAP [22] & 2017 & Power efficient & Construction of optimal spanning trees \\
\hline $\begin{array}{l}\text { WPO-EECRP } \\
\text { [23] }\end{array}$ & 2017 & $\begin{array}{l}\text { Considers trust and energy for } \mathrm{CH} \\
\text { selection }\end{array}$ & $\begin{array}{l}\text { Moderate degree of energy } \\
\text { imbalance exists }\end{array}$ \\
\hline DUCR [24] & 2017 & Deals with hotspot problem & The clusters are not of equal size. \\
\hline EECS [25] & 2018 & Balanced clustering & Energy unstable \\
\hline EA-CRP [26] & 2018 & Planned deployment & $\begin{array}{l}\text { Too many control messages can drain } \\
\text { network life }\end{array}$ \\
\hline BEERP [27] & 2018 & $\begin{array}{l}\text { Uniform load for maximum of the } \\
\text { sensor nodes }\end{array}$ & $\begin{array}{l}\text { Poor in multilevel heterogeneous } \\
\text { network }\end{array}$ \\
\hline ICFSFDP [28] & 2018 & $\begin{array}{l}\text { Potential } \mathrm{CH} \text { selection through trust } \\
\text { computation }\end{array}$ & Imbalance in energy \\
\hline ECCRP [29] & 2018 & Prevents the issue of energy hole & $\begin{array}{l}\text { Trust and energy are not } \\
\text { optimized }\end{array}$ \\
\hline $\begin{array}{l}\text { IACO- } \\
\text { PEGASIS [30] }\end{array}$ & 2018 & Handles the rotation of $\mathrm{CH}$ & Energy is not optimized \\
\hline TEEN [31] & 2019 & Improve lifetime & Lack of communication \\
\hline APTEEN [32] & 2019 & Data aggregation is periodic & Processing capability is slow \\
\hline DEAR [33] & 2019 & Routing paths are known prior & Transmission delay \\
\hline BPA-CRP [34] & 2019 & It considers network topology & $\mathrm{CHs}$ are elected randomly \\
\hline $\begin{array}{l}\text { Bkd-TREE } \\
{[35]}\end{array}$ & 2020 & $\begin{array}{l}\text { Significant method cluster } \\
\text { formation and management }\end{array}$ & $\begin{array}{l}\text { There is room for energy stability and } \\
\text { network lifetime }\end{array}$ \\
\hline ECRP [36] & 2020 & Cluster setup done only once & $\begin{array}{l}\text { Some sensor nodes are left without } \\
\text { cluster heads }\end{array}$ \\
\hline
\end{tabular}




\section{Factors that drain out energy}

G a o et al. [38] provided two reasons why energy efficiency is regarded as a major issue in WSN: (i) Resource constraints; (ii) Applications in which WSN is used. The deployment requirements of a WSN will differ depending on the application. In this case, the network's lifetime is defined as the time elapsed between the sensing function and the data transfer to the sink. As a result, because the sensor range is wider than the energy consumed, the sensor range must be limited in order to minimize the use of energy in a network, according to A mutha, Sharma and $\mathrm{Nagar}$ [39]. In this section, we will focus on four major WSN factors that can influence energy consumption.

1. Radio module. According to Aleksejs Jurenoks and Novickis [40], the radio module can be in four states: inactive, sleeping, transmitting and receiving, and transmitting and receiving. The distinction between the sleep and idle states is that when the node is in the sleep state, it is considered to be off, whereas when the node cannot transmit or receive, it is in the idle state. Both states are most likely to result in the abolition of energy consumption. According to $\mathrm{Cu} \mathrm{i}$, Goldsmith and Bahai [41], when the distance between nodes is too great, the energy consumption increases, and the transmission time eventually increases. When errors are found per bit, J ung, W ang and In gram [42] use the expression Bit Error Rate to reduce the transmitting time. Modulation schemes can be used to shorten the distance between nodes. Similarly, collaborative communication techniques can improve signal efficacy. According to J a y aw e e r a [43], Single Input Single Output (SISO) and Multiple Input Multiple Output (MIMO) systems consume less energy and have shorter transmission power delays. The power transmission management technique aids in the monitoring of delays, inference, communication, and connectivity performance. $\mathrm{G}$ a o et al. [38] employ Cooperative Topology Adaptation Control (CTCA), which is a device that employs directional antennas to improve transmission range and throughput. These directions will also improve the network's capacity and life cycle, resulting in low power, low interference, and a wide range of low bands.

2. Data reduction. Amutha, Sharma and Nagar [39] argue that the amount of data sent to the sink must be reduced. This is referred to as data reduction. The data is combined and forwarded to the sink to reduce network congestion and load. We use data compression techniques to optimize transfer rate and time to improve energy efficiency. According to $\mathrm{Cunji}$ a n g Y u [44], this can reduce the size of the packet during transmission.

3. Sleep and Wakeup systems. R e $\mathrm{n} n$ ing $\mathrm{X}$ i e, Li u and G a o [45] mention that nodes are put to sleep when they are not allowed to participate in data aggregation and data sharing. To send or receive messages, the nodes that are sleeping must wake up, and we thus follow a work loop phase that will change the node states Xiaoying Yang and Zhang [46]. While this appears to be a time-saving strategy, it wastes time during redundant wake-up schemes. Aleksejs $\mathrm{J}$ u re n o k s and $\mathrm{N} \mathrm{ov}$ ick is [40] claim that when assigned low duty cycles, energy consumption increases as delays increase. Low-powered radios only serve to wake up a node when packets are sent or received. 
4. Battery depletion. To monitor the current power in the WSN, early prediction mechanisms are required. V lad $\mathrm{m}$ ir $\mathrm{Sh}$ ak o v and K o o [47], reduced power consumption by adjusting parameters such as service cycle, propagation range, and sampling rate. When the battery leakage occurs, the network's reliability will be unknown. Vladmir Shakov and Koo [47] propose another method for charging the battery, which is typically accomplished through magnetic resonance and electromagnetic radiation. It must be combined with low detection and power requirements through the use of electromagnetic radiation. Magnetic resonant connectors, on the other hand, within a few meters of each other, address energy efficiency. When energy is exchanged with neighbouring wireless network charging nodes, it becomes a challenge, according to Gnanaprasambikai and Munnusamy [48]. Recent advancements in wireless technology allocate a charging vehicle to each cluster in the WSN, and the path to be travelled is determined by electricity. A muth a, Sh a r m a and $\mathrm{Nag}$ a r [39], propose using mobile robots to recharge sensor batteries. A device called WINCH is used, and this model is designed in such a way that it can quantify the energy obtained by each sensor. A recent advancement is the use of multimode wireless networking technology and wireless charging vehicles on the basis of a mobile system in which the twodimensional plane is divided into hexagonal cells.

\section{Performance metrics}

According to Ezhilarasi and Krishnaveni [1] the overall performance of the network must be evaluated using specific metrics because problems such as collisions, overhearing, protocol overhead, and idle listening are a hindrance to better WSN performance. This section discusses the metrics that must be considered in order to improve the network's energy efficiency and survival.

Energy consumption. It is the difference between the initial energy before transmission and the energy after transmission

$$
E_{\mathrm{c}}=E_{\mathrm{i}}-E_{\mathrm{f}}
$$

where $E_{\mathrm{c}}$ is the energy consumed, $E_{\mathrm{i}}$ is the initial energy and $E_{\mathrm{f}}$ is the final energy.

Ratio of packet delivery. The packets start transmitting from the source and complete at the destination. The ratio between the numbers of packets reaching the destination to the ratio of the number of packets sent with a constant speed by the source is defined by

$$
P_{\mathrm{d}}=\left(\frac{P_{\mathrm{r}}}{P_{\mathrm{s}}}\right) \times T_{\mathrm{st}}
$$

where $P_{\mathrm{d}}$ is the packet delivery ratio, $P_{\mathrm{r}}$ is the number of packets received, $P_{\mathrm{s}}$ is the number of packets sent, and $T_{\text {st }}$ is the speed of transmission.

End-to-End Delay. It is the time used by the packet for transmission between the source and the destination. Delays triggered by road bridges, tailings, retransmission, distribution and transfer of data packets may be included in this journey time

$$
e_{\mathrm{d}}=\frac{T_{\mathrm{end}}-T_{\mathrm{start}}}{P}
$$


where $e_{\mathrm{d}}$ is the End-to-End delay, $T_{\text {end }}$ is the time when the packet arrives at the destination, $T_{\text {start }}$ is the time when the packet started its transmission from the source and $P$ is the total number of packets.

Communication overhead. The sum of packets from one sensor node to the other is given as the number of packets being transmitted. The overhead touch may involve the head of the routing table, the routing system and the packet structure in the sensor nodes

$$
C_{\mathrm{o}}=\left(\frac{P_{1}}{P_{\mathrm{r}}}\right) \times 100,
$$

where $C_{\mathrm{o}}$ is the communication overhead $P_{\mathrm{l}}$ is the number of packets lost and $P_{\mathrm{r}}$ is the number of packets received at the destination.

Throughput. It is the number of transmitted packets per unit time. This is the ratio of total number of packets to the number of packets that have been sent. The efficiency is in bits per second (bps) or in packets per second (pps) is calculated

$$
\mu=\frac{P_{\mathrm{S}}}{P},
$$

where $\mu$ is the throughput in s, $P_{\mathrm{s}}$ is the number of packets sent, and $P$ is the total number of packets.

Misbehaviour ratio. It is defined as the number of packets affected by wormhole attacks to the total number of packets sent

$$
P_{\mathrm{m}}=\frac{P_{\mathrm{a}}}{P_{\mathrm{s}}},
$$

where $P_{\mathrm{m}}$ is the misbehavior ratio, $P_{\mathrm{a}}$ is the number of packets attacked, and $P_{\mathrm{s}}$ is the number of packets sent.

Connectivity ratio. It is defined as the ratio of weak connections to overall network connections

$$
C_{\mathrm{c}}=\frac{C_{\mathrm{w}}}{C_{\mathrm{n}}},
$$

where $C_{\mathrm{c}}$ is the connectivity ratio, $C_{\mathrm{w}}$ is the number of weak connections, and $C_{\mathrm{n}}$ is the total number of connections in the network.

Packet loss. It is defined as the number of packets dropped to the number of packets sent

$$
P_{1}=\left(\frac{P_{\mathrm{drop}}}{P_{\mathrm{s}}}\right) \times 100,
$$

where $P_{1}$ is the packet loss, $P_{\mathrm{drop}}$ is the number of packets dropped, and $P_{\mathrm{S}}$ is the number of packets sent.

\section{Mac protocol approach}

The architecture of WSN MAC protocols may be an important aspect of energy efficiency. Energy savings in a WSN can be easily attained by designing efficient MAC protocols. WSN MAC protocols are concerned with energy consumption as well as network adaptability, reliability, and scalability states Farhan a A froz and Braun [49]. According to Indra Shah, Maity and Dohare [50], MAC protocols are due to radio functionalities such as idle listening, collisions, overhearing, control packet overhead, sleep/active period, and data overflow in the network. The MAC layer governs how sensor nodes transmit and receive data, as 
well as how sensor nodes compete for medium access. The MAC's primary function is to restrict access to the medium by one or more interfacing nodes at the same time in order to avoid collision and ensure that shared-medium network work is successful stated $\mathrm{S} \mathrm{m}$ it a et al. [51]. Hu a $\mathrm{g}$ et al. [52] divide MAC protocols into synchronous and asynchronous energy efficiency categories. The synchronous party wakes up nodes that rely on rendezvous and skewed categories. One of the most effective methods for reducing energy consumption in WSN is duty cycling. V anes s a $\mathrm{Q}$ u in t e ro et al. [53] offer a variety of energy-saving techniques, including energyefficient schedules, service schedules, on-demand wake-up plans, and so on. V aness a Quintero et al. [53] explain that during appointment, all nodes wake up and sleep at the same time, and synchronization occurs in stages. R e n n in $\mathrm{g} X$ i e, $\mathrm{Liu}$ and Gao [45], propose duty-cycling, which involves changing the responsibility for the node based on time-space problems in order to reduce collision rates and define controls. The decision-making process for energy production is also affected by battery status.

Farhana Afroz and Braun [49] clearly discuss how MAC protocols are classified into three types: TDMA-based, contention-based, and hybrid. Contentionbased MAC protocols are further subdivided into synchronous and asynchronous protocols. TDMA-based MAC protocols are primarily a subset of reservation-based MAC protocols. Time is divided into frames in TDMA-based MAC, and each frame is further subdivided into a number of slots. Each sensor node is assigned a specific guaranteed time slot during which it can transmit or receive data. At other times, it turns off its radio. As a result, scheduling-based MAC protocols allow for collisionfree transmission and can improve throughput, but at the expense of synchronization overhead. Another issue with TDMA-based MAC schemes is that they provide poor channel utilization when only a few sensor nodes want to transmit data. There are two types of TDMA-based MAC protocols: centralized and distributed schemes. The base station or Cluster Heads $(\mathrm{CH})$ allocate time slots to all nodes within the WSN in a centralized TDMA-based MAC protocol. The slot assignment, on the other hand, is not managed by any centralized entity in the distributed MAC protocol. Instead, the nodes can control their schedules based on information from the local network. CSMA is the foundation of contention-based MAC protocols. They listen to the channel before sending the data. It tries to save energy by controlling the operational activity of sensor nodes. Duty cycling, in which a node switches between active and sleep modes, is one mechanism used for this. When a sensor node is active, it consumes a significant amount of battery power. As a result, in order to save energy, the nodes should be in active mode for the shortest amount of time possible. This can be accomplished through low duty cycling, but this technique increases end-to-end delay. Synchronous MAC protocols set wake time on a regular basis so that data can be transmitted while in active mode, but this can result in additional synchronization overhead. Asynchronous MAC protocols allow sensor nodes to set their own wakeup schedule, removing the need for synchronization, but it requires close coordination among the nodes. Asynchronous MAC is further classified as MAC with one radio and MAC with Wake up Radio (WuR). A node in MAC with one radio begins sending preamble, whereas all sensor nodes in MAC with Wake up Radio remain in 
sleep mode. The WuR keeps an eye on the channel. When a node needs to send a message to a neighbor, it starts by sending a short wake message to its neighbors. Hybrid MAC protocols are a fusion of TDMA and CSMA, each with its own set of limitations. A few MAC protocols used in WSNs are listed below:

1. S-MAC (Sensor MAC-2002). It is a synchronous MAC protocol that provides collision avoidance and good stability states while consuming less energy state Ye, Heidemann and Estrin [54]. Long messages are divided into segments and forwarded in burst format using this protocol. It is divided into three modules: collision avoidance, periodic listen/sleep, and message passing. It reduces the nodes' listen time and periodically puts them to sleep. Time is divided into frames, and each frame is subdivided into three periods: SYNC, DATA, and SLEEP. Each node has a predefined wake and sleep time. At the start of the SYNC period, all nodes in a cluster wake up to get their clocks synchronized with one another. During the DATA period, multiple nodes with data packets compete for the medium. Nodes that do not have any data packets go to sleep at the start of the SLEEP period. After completing data transmission, other nodes will turn off their radio. It has very good energy-saving characteristics, but suffers from end-to-end latency caused by periodic sleeping, especially when there is a lot of network traffic.

2. STEM (Sparse Topology Energy Management-2002). It is an asynchronous multi-hop protocol proposed by $\mathrm{Schurgers}$ et al. [55] that uses separate channels for the main radio and $\mathrm{WuR}$, avoiding interference between wake up message and data. STEM has two variants based on the format of the wake-up message, STEM-B and STEM-T. STEM-B initiates transmission by sending wakeup beacons with the sender and receiver MAC addresses until the beacon receives an acknowledgement from the targeted radio. Following receipt of the acknowledgement, the sender and receiver activate their primary radios for data transmission. WuR is also looking for wake-up messages from other nodes. STEMT performs similar to STEM-B but a simple tone is transmitted instead of transmitting a beacon. The disadvantage of STEM-T is that a simple tone can wake up all of the neighboring nodes, increasing power consumption. Because the wake-up tone must be sent for an extended period of time, the delay increases.

3. T-MAC (Timeout MAC-2003). V an Dam and Langendoen [56] propose this synchronous MAC protocol to reduce energy consumption caused by idle listening. To reduce energy consumption, an adaptive duty cycling approach was suggested here. The idle listening and sleep period in TMAC ends when no activation event occurs for the specified time duration. This is the shortest amount of time allotted for idle listening before the nodes go to sleep. Because TMAC has been identified as having an early sleeping problem, a mechanism known as FutureRequest-To-Send (FRTS) is proposed to overcome it. A node can use this mechanism to notify its target that it is unable to access the channel at the specified time. This protocol improves network adaptability and performance in heterogeneous networks. It does, however, have a synchronization issue.

4. TRAMA (Traditional Assisted MAC-2003). R a j e $\mathrm{d}$ r a n, O bra c z i a and Garcia-Luna-Aceves [57] suggest it. It is a TDMA-based distributed protocol in which time is divided into slots, and each slot is further divided into 
random access intervals and data transmission intervals. Nodes broadcast their transmission schedule to their one hop neighbors on a regular basis and learn about their two hop neighbors.

5, D-MAC (Dynamic MAC-2004). Lu, Krishnamachari and $\mathrm{R}$ a g a v e $\mathrm{n}$ d a [58] propose it as a low-latency MAC protocol designed specifically for converge cast communication. This protocol attempts to alleviate the problem of data forwarding interruptions. This issue occurs when sensor nodes on a multi-hop path to the sink are unaware of the ongoing data transmission and go to sleep until the next cycle, resulting in significant sleep delay for packet delivery. DMAC employs the data gathering tree concept to enable continuous packet forwarding. To solve the sleep delay problem, the data prediction method is used. When same-level nodes in the tree compete for access to the medium, the More-to-Send mechanism is used. DMAC offers remarkable energy savings and low latency while ensuring high data delivery reliability. The disadvantage of this protocol is that it does not support collision avoidance, so collisions occur when multiple nodes with the same schedule attempt to transmit to the same node. It is also impossible to form a data gathering tree if the data transmission paths are unknown in advance.

6. BMA (Bit Map Assisted MAC protocol). It is a cluster-based protocol proposed by Li and L a zar ou [59] that is based on traditional TDMA. BMA is an event-driven intra-cluster communication protocol. This protocol's operation is divided into rounds, with each round having two phases: setup and steady state. During the setup phase, the WSN is divided into clusters, with the Cluster Head being the node with the most energy in each cluster. The steady state phase is divided into sessions, each with a fixed duration and a contention period followed by a data transmission or idle period. Because all nodes are not required to send data in a single session, the data transmission period is variable.

7. B-MAC (2004). It is an asynchronous CSMA protocol proposed by Polastre, Hill and $\mathrm{Cu} 1 \mathrm{ler}$ [60] that focuses on high channel utilization, collision avoidance, and low power operation. It employs adaptive preamble sampling to reduce duty cycling and idle listening while also tolerating changing network conditions. It employs a clear channel assessment mechanism to determine whether or not there is any activity in the medium, after which a node enters Low Power Listening (LPL) mode. When a node wakes up, it listens to the long preamble to determine whether it should stay awake or go to sleep. This method saves a significant amount of energy. The flexibility of BMAC allows for higher throughput, higher packet delivery rates, less elapsed time, and less energy consumption.

8. RATE-EST (Rate Estimation MAC-2004). Mille r and Vai dy a [61] propose a traffic adaptive multi-hop wake up MAC protocol. It dynamically adjusts subsequent wake up time based on packet arrival rate. The main channel is used for control and data packet transmission, while the wake-up channel is used to wake up neighboring nodes. Low duty-cycled wake up radios help to reduce power consumption, idle listening, and improve latency to wake up neighbors. To control the delay, a queue threshold is defined. When the queue reaches its limit, the sensor node's WuR begins sending busy tones, causing all neighbors to go into full wakeup mode. All full wake-up nodes turn on their main radio and listen to their main 
channel until a filter packet arrives. A filter packet tells which node to keep its radio on for reception while the other nodes go back to sleep to save energy. The disadvantage of this protocol is that the full wake-up mechanism is expensive, so a triggered wake-up mechanism is used instead, in which the source node calculates its data transmission rate and attempts to schedule a triggered wake up with its destination.

9. Wise-MAC (2005). E 1-H o i y di and D e c o ti g n i e [62] propose an Alohabased wireless sensor MAC protocol to reduce energy consumption. It uses a preamble sampling method to ensure low power consumption in WSNs. The medium is sampled on a regular basis to monitor activity. If the medium is busy, the sensor will continue to listen until it finds it idle. Every data frame includes a wake-up preamble to ensure that the receiver is awake when the data arrives. The data acknowledgement sent by the receiver includes the next wake up time schedule. The transmitter can learn the specifics of the receiver's wake-up and schedule transmission accordingly. Overhearing and latency are reduced as a result. Though it addresses several issues related to low power WSNs, it does not provide any mechanism for sensor nodes to adapt to changing traffic conditions.

10. TEEM (Traffic-aware Energy Efficient MAC-2005). $\mathrm{S} \mathrm{u} \mathrm{h}$ and Co [63] propose to reduce it to idle listening time and control packets. The duty cycle must be updated on a regular basis. It has a high chance of being overheard, and the latency is extremely high.

11. X-MAC (2006). Buettner et al. [64] propose this protocol to achieve goals by overcoming the challenges of low power listening. XMAC divides the long preamble into a stream of short preamble packets, each of which contains the target node ID. When a node receives a short preamble packet during its wake-up period, it checks to see if the target node's ID in the packet matches its own ID. If it is the desired receiver, it remains awake for incoming data; otherwise, it falls asleep. $\mathrm{XMAC}$ is notable for inserting small pauses between short preamble packets, allowing the sender to pause and listen to the medium. The receiving node can send acknowledgement to the sender during the pause. When the transmitter receives acknowledgement, it stops sending preambles and starts sending data. Traditional low power listening schemes have been shown to outperform XMAC.

12. A-MAC (Adaptive MAC-2007). $\mathrm{N}$ a m, L e e and $\mathrm{J} \mathrm{ung} \mathrm{[65]} \mathrm{propose} \mathrm{it,}$ which uses sink-triggered route discovery for new routes while reducing unnecessary control overhead. It considers residual energy to improve network performance, so it increases delay and consumes more energy.

13. R-MAC (Routing Enhanced MAC-2007). D u, S a ha and J o h n s o n [66] propose it, and it uses cross-layer routing information to reduce latency and improve contention handling while limiting energy efficiency and throughput. It employs a set of control frames known as PIONeer frames (PIONs) that are used across multiple hops. The PIONs make a communication request and wait for confirmation. PION has its own address as well as previous and next hop addresses. Using the PION mechanism can add to the complexity of handling packets.

14. EA-TDMA (Energy Adaptive TDMA-2008). Shafiullah et al. [67] propose it as a TDMA-based protocol. It overcomes energy dissipation through idle 
listening, allowing sensor nodes to enter a sleep state if there is no data for transmission in its assigned slot. It saves energy due to idle listening, but the Cluster Head must be active at all times.

15. Y-MAC (2008). $\mathrm{K}$ i $\mathrm{m}, \mathrm{S} \mathrm{h}$ in and $\mathrm{C} \mathrm{h}$ a [68] propose a variant of the TDMA protocol. It is very efficient for low-traffic networks and works with multiple channels. Frames and slots are used to divide time. A broadcast period and a unicast period are combined to form each frame. Every node is active and competing for medium access at the start of a broadcasting period. At the beginning of each slot, the nodes that want to send data compete for the channel in the contention window. The node that wins the competition is the one who can send data.

16. RI-MAC (Receiver Initiated MAC-2008). S u n, Gurewitz and J o hn s o n [69] propose it to handle efficiently a wide range of network traffic. It reduces latency by reducing the amount of time the sender and receiver occupy the channel to exchange data. According to their duty cycle schedule, all nodes wake up on a regular basis. If the receiver discovers that the medium is idle after waking up, it sends a short beacon frame to the sender to notify it when to begin data transmission. The sender immediately sends the data after receiving the beacon. After completing data transmission, the sender goes back to sleep. If the sender does not respond after receiving the beacon, the receiver goes to sleep. In this case, the sender must keep its radio on until the receiver enters wake-up mode.

17. O-MAC (Optimized MAC-2008). It was proposed by Y a d a v, V a r ma and Malavi y a [70], and it adjusts the duty cycle based on traffic load, increasing the possibility of latency and energy consumption.

18. W-MAC (Wave MAC-2009). $\mathrm{N}$ i a f a $\mathrm{r}$ and $\mathrm{S}$ h a h h o s e in i [71] propose it to improve energy efficiency in wireless sensor networks. Because the network is deviated into concentric areas, a good localization algorithm is required. However, it reduces the overhead caused by control packets as well as the end-to-end delay.

19. C-MAC (Convergent MAC-2009). It is an asynchronous contention MAC protocol proposed by Liu, Fan and Sinha [72]. When there is no traffic to transmit, it uses asynchronous sleep scheduling. It wakes up potential forwarders with anycast and then gradually converges anycast to unicast with synchronized scheduling, removing anycast overhead. To save power after convergence, a C-MAC variant, C-MAC-S, was used. After convergence, C-MAC-S employs staggered scheduling.

20. DC-MAC (Duty Channel-based MAC-2010). Z h e ng et al. [73] propose implementing a periodic sleeping/listening method in conjunction with synchronization to save energy in multi-hop scenarios. When a node has data to transmit, it turns on its main radio and WuR to listen. When the channel is free, it sends a busy tone to wake up its neighbors, and neighbors within one hop of the busy tone listen to it and activate their main radios. A mechanism is used to select the best relay nodes in order to avoid collisions and minimize delay. Although DC-MAC reduces latency and saves energy, it adds computational complexity.

21. PW-MAC (Predictive Wake-up MAC-2011). T a ng et al. [74] propose an asynchronous MAC protocol. The sender is enabled in this protocol to predict the receiver's wake up time, and the sender also turns on its main radio before the 
receiver wakes up. It controls the wake-up time of each node using a pseudo-random sequence. It lowers the duty cycle of the sender and receiver, allowing for more accurate predictions. To improve reliability, PW-MAC employs an on-demand prediction error and correction mechanism. To avoid collisions in the channel, the channel prediction-based retransmission mechanism is used. This protocol adds extra overhead due to beacon messages and idle listening.

22. SA-RI MAC (Sender Assisted-Receiver Initiated MAC-2011). H e n n a [75] propose it based on an asynchronous mechanism. To begin transmission, a sender waits for a specific beacon from the receiver. The sender uses the channel access failure counter to track the number of failures encountered while accessing the channel while sending data to the receiver. The counter value is exchanged at regular intervals between competing senders via a specific beacon. The receiver in the beacon defines a back-off window size value to estimate the contention level. If the value exceeds the maximum contention window, high contention is indicated, and the sender compares its channel access value to the counter values of its neighbors. The node with the most attempts to contact the receiver is given the highest priority for data transmission. If the sender detects a competing neighbor, it turns off its radio to save energy and reduce contention at the receiver.

23. DCW-MAC (Duty Cycled Wake-up receiver MAC-2011). M a z l o u m and Edfors [76] propose that an effective duty cycle mechanism be used to minimize energy consumption. A sleep/listen timer controls it, and it periodically scans the channel for a wake-up beacon. When there is data to be received, the main receiver is turned on. When the sender has data to send, it activates the main radio and transmits a beacon to the target. When a beacon with the sender ID is detected, the receiver sends acknowledgement to the sender. When the sender receives the acknowledgement, the transmission process begins. Following receipt of the data, the receiver sends an acknowledgement.

24. CL-MAC (Cross Layer MAC-2013). He f e i d a, C a n li and $\mathrm{K} h$ o $\mathrm{k} \mathrm{h}$ a r [77] propose a method in which a single node can schedule multiple flows for multiple destinations, resulting in reduced latency and increased throughput. The length of the flow setup packets is long, increasing the network's energy consumption.

25. AS-MAC (2013). Jang, Lim and Sichitiu [78] first propose it. The sensor nodes in this protocol minimize the preamble length by storing the wake-up schedule of neighboring nodes. It is divided into two phases: initialization and sleep. Nodes build neighbor tables and store scheduling information for their neighbors during the initialization phase. It selects and broadcasts periodic wakeups based on this information. The disadvantages are the overhead associated with broadcasting and the requirement to store a one-hop neighbor table.

26. WX-MAC (2013). $\mathrm{H}$ a n et al. [79] propose it, with the goal of shortening the preamble length and keeping the sensor nodes in the wake-up state as short as possible. Sensor nodes communicate their sampling schedules using report and query mechanisms. The nodes estimate the precise time of initiation of the preamble. It supports the strobe preamble approach, which allows it to stop preamble transmission, start data transmission, and return to sleep at the appropriate time. 
27. SW-MAC (Sleep Window MAC-2014). It was proposed by Li a ng et al. [80] based on network traffic patterns. To reduce energy consumption, the sleep window was adjusted based on traffic patterns, and a scout-based scheduling mechanism was implemented. The receiving node estimates the arrival time of the traffic and dynamically adjusts the sleep window based on the varying traffic loads. At regular intervals, each node wakes up and listens to the channel for a scout packet. When a scout packet is detected, the receiver responds by sending an acknowledgement to the sender. When the sender receives acknowledgement, it stops sending scout packets and begins transmitting data. The receiving node will stay active in order to receive data packets and forward them to the next hop. The active time of a node is determined by the wireless channel's bandwidth, the length of the scout packet, the queue length, and the number of sending nodes. A node's sleep time is kept as long as possible until a packet is received. When a node receives a packet, it reduces its sleep time in proportion to the packet's waiting time. The sleep window is controlled by the estimated sleep time. This method reduces end-to-end delivery latency.

28. TR-MAC (Transmitted Reference MAC-2014). Morshed and $\mathrm{He}$ ij e $\mathrm{nk}$ [81] propose it as an asynchronous MAC protocol. The sender first sends a small preamble and then waits for the receiver's acknowledgement. The transmitter can reduce the length of the preamble to save energy. The receiver conserves energy by remaining in sleep mode the majority of the time. The receiver only wakes up to detect channel activity. It reduces energy consumption from four major sources: idle listening, collision, overhearing, and overhead.

29. TAS-MAC (Traffic Adaptive Synchronous MAC-2016). Li u, H u a n g and $\mathrm{Xi}$ a o [82] propose it to achieve low delay, high throughput, and low energy consumption. Duty-cycling reduces energy consumption while also limiting throughput and increasing end-to-end latency. TAS-MAC assigns time slots only to contending nodes that are active routes, ensuring high throughput. It achieves low end-to-end latency by informing all active-route nodes in advance of incoming data traffic.

30. AS2-MAC (2016). An ch or a et al. [83] propose using a smart awake approach to reduce energy consumption. During the initial network configuration phase, each node creates a map of its neighbors that includes information about their wake-up times. Each neighboring node has an entry in the wake-up table. During the receiving phase, each node uses the data collected in the wake-up table to turn on its radio only when it has data to transmit or receive from its neighbors. It reduces unnecessary node wake-up, resulting in lower energy consumption. It employs a lowpower MAC mode known as coordinated sampled listening to check for incoming transmissions on the channel on a regular basis. If the chosen packet rate is consistent with the network's bandwidth, this protocol performs better in terms of energy efficiency and packet delivery ratio.

31. MMSMAC (Multi-Mode Sensor MAC-2017). U sha, Has sen and $\mathrm{S}$ a h a [84] propose it, and it works in three modes: synchronous, asynchronous, and hybrid. It outperforms in terms of latency and energy consumption. 
32. PRIN-MAC (Priority-In-Node MAC-2017). Subramani and Paramasivam [85] propose it as a QoS-based MAC protocol. It uses static priority for network nodes, allowing them to reach the sink node in a single hop.

33. ADC-MAC (Adaptive Data Collection MAC-2017). T ong and Pan [86] propose that free addressing and dynamic duty cycling improve load adaptability and energy efficiency in wireless sensor networks. This protocol ensures that any two communicating nodes with two adjacent grades will not be interfered with by their previous/next grade neighbor, but interference with the same grade neighbor is possible.

34. DBDDCA (Distance-Based Dynamic Duty-Cycle Allocation-2019). It was proposed by In d r a K m ar S hah [50] to assign dynamic duty cycle to the nodes in the network. A duty cycle is assigned to the child node, according to their distance from the $\mathrm{CH}$. As $\mathrm{CH}$ changes each round the same child node has a different distance for different CHs. The duty cycle allocation for the same child node will vary each round. DBDDCA saves power consumption by the dynamic allocation of duty cycle and therefore packet delivery is high. The energy consumed for transmission to longer distance nodes is less. The initial energy consumption is less but when the number of rounds increases energy consumption also increases. This shows high utilization of network energy. The network is assumed dead when no transmission or reception of signal occurs in the entire network. The network is assumed dead when no transmission or reception of signal occurs in the entire network.

35. DET-MAC (2020). Gu ls han and Kand as amy [87] propose it as a solution to the early sleep problem. This protocol has eight MAC states. It constrains the extension of the active period so that the packets sent by the source node are limited, giving the relay nodes more opportunities to forward packets to the sink node. It also consumes less energy because the active period in nodes is shorter, which can increase network lifetime.

\section{Load balancing}

Load balancing is a technique for equalizing the energy consumption of all nodes, allowing all nodes to degrade at the same time. Efficient energy consumption and load balancing are critical in wireless sensor networks for increasing network lifetime, packet delivery ratio, and end-to-end delay discussed $\mathrm{Sneha}$ and $\mathrm{Nagarajan} \mathrm{[88].} \mathrm{The} \mathrm{lifetime} \mathrm{of} \mathrm{the} \mathrm{network} \mathrm{is} \mathrm{increased} \mathrm{by} \mathrm{load} \mathrm{balancing}$ because it is not dependent on the life of the weak node but on the life of all the nodes in the network. A large number of load balancing techniques and algorithms have recently been proposed in WSN, and we will only discuss a few of them in this section.

Gupta and Younis [89] improve the cluster member selection by employing a comprehensive weight value comprised of the distance between the cluster head and the member as well as the residual energy. He also employs an optimization threshold value to avoid load imbalance. The algorithm considers load equalization when developing the balanced cluster. $\mathrm{M}$ a and $\mathrm{Y}$ an $\mathrm{g}$ [90] propose an 
algorithm in this study that accounts for the problem of positioning mobile cluster heads and balancing traffic load in a hybrid sensor network with static and mobile nodes. It has been demonstrated that the location of the cluster head can have a significant impact on network lifetime. The network load can be balanced and the cluster head's lifetime increased by moving it to a better location. In Low et al. [91] density is taken as a key parameter and proposes a load-balancing algorithm for cluster heads in wireless sensor networks that takes traffic load into account. The traffic load supplemented by entire sensor nodes is assumed to be the same, which is the special case of this algorithm. It's an NP-hard problem. It employs a centralized approach and assumes that all nodes are aware of the network. D e $\mathrm{ng}$ and $\mathrm{Hu}$ [92] propose load balanced group clustering to balance battery power by implementing dynamic route calculation based on the network's energy distribution condition.

$\mathrm{Ch}$ en $\mathrm{g}$ and $\mathrm{Yu}$ [93] combine TSP-Reduce algorithm finds each overlapping point as the shortest path in the network because the total path between the source and destination was too long, increasing communication costs. S h a r m a, Y o u and $\mathrm{K} \mathrm{u} \mathrm{m}$ a r [94] propose data dissemination scheme addressed load balancing in UAV coordinated WSNs. To achieve energy efficiency, it used a firefly optimization algorithm. Li u et al. [95] propose light-path provisioning technique is used in the network to achieve load balancing in terms of scheduling modulation level and traffic management. Singh, Toor and Jain [96] propose Analytical model for load balancing selects the best path from source to destination based on the energy consumption of ordinary nodes. Maio et al. [97] propose multi-hop relay communication and multipath weighted revenue algorithm for load balancing updates route information with data collected from participating nodes to balance load in the network. Fang et al. [98] proposed a software-defined method for load balancing that used software configuration to transform ordinary sensor devices into smart devices that can manage their tasks based on residual energy. Although it performed well in terms of energy conservation, the network's deployment was complicated. Lubin Balasubramanian and Govind as a m y [99] use Enhanced Hybrid Multipath Routing (EHMR), a hierarchical multipath approach that incorporates inherent clustering and single load balancing. It employs multiple paths with ondemand load balancing, allowing the entire load to be transferred to another path in the event of a route failure. In this approach, sensor nodes are deployed at random and assigned to a specific transmission range. This approach employs seven distinct, interconnected algorithms to achieve load balancing while consuming the least amount of energy. Gherbi, Aliouat and B e n moh a mmed [100] Distributed Energy-efficient Adaptive Clustering Protocol (DEACP) has load balancing and selfadaptation capabilities. This protocol is effective in reducing overall network energy consumption as well as balancing energy consumption between sensors and, as a result, prolongs the lifetime of the network. Ezhilarasi and Krishnaveni [101] propose EMEER algorithm contributes to load balancing by partitioning the entire network into clusters using conventional clustering and selecting the optimal $\mathrm{CHs}$ using the cuckoo search algorithm. Depending on the condition of the sensing terminal node, the nodes can be in one of four states. When data arrives at the terminal end, the node switches from sleep to transmit mode, and after transmitting sensed 
information to the forwarding node, it returns to idle mode, consuming significantly less power than in the transmit mode. El Ala mi and Najid [102] Enhanced Clustering Hierarchy emploied a sleep and wake-up mechanism for adjacent nodes in a WSN to reduce energy consumption of participating nodes and extend network lifetime. Li et al. [103] proposed Energy-Efficient Load Balancing Ant-based Routing Algorithm (EBAR) resolves load balancing in WSNs. To achieve load balancing, this method used an improved pheromone trial and a pseudo random route. It adheres to greedy expected energy metrics in order to reduce energy consumption by route establishment. B e n et al. [104] propose energy efficient load balancing scheme uses path distance information from participating nodes to achieve low energy transmissions with optimal latency. S u n et al. [105] propose hierarchical data job scheduling dynamically manages the priority of task scheduling in the network to avoid task starvation and maximizes system output with optimal resource utilization. W ang's et al. [106] task distribution technique for load balancing employs a heuristic algorithm to schedule tasks in parallel in the deployed WSN environment. To reduce energy consumption, they use the decision-making process of wireless nodes. Alagmir Naushad et al. [107] propose the Neighbour Discovery algorithm, which is designed to identify neighbour sensors and adjust their hop count to the base station, as well as the Hop Number Update algorithm, in which the base station updates the hop number and retransmits the HU packet to the nodes. He also used another algorithm, the Path Request, which recognizes the various communication routes between neighbours. A l a g m i r N a u s had et al. [107] also propose the next Maximum Energy and Minimum Hop algorithm, which determines the main path with the most residual energy but the shortest distance from the hop. S mriti S achan et al. [108] use EHMR, which proved to be energy efficient, balanced, and load efficient. As the network density increases, so does the efficiency of EHMR. In multi-hop networks, there is a mechanism for static connections. For multi-hop networks, there is a static connection solution for relaying data from sensor nodes to the base station. There is a chance that data packets will be streamed across the network in this case. A y a n Panja and Gh os h [109] achieve load balancing in a WSN using standard techniques such as Breadth First Search and Dijkstra's algorithm, which finds the shortest path to route data packets. The central principle of load balancing is to reduce hot spots in order to extend the network's life. Pri in c e R ajp o ot and D wivedi [110] introduce a heuristic algorithm that focuses on the transmit power of the data transmission route to equalize the load, ensuring that each node in the network receives the same transmission power. To improve the network's existence, the non-linear programming approach is updated based on the WSN topology and traffic propagation through the network. A research paper introduces the concept of control in order to reduce link usage by configuring a network controller to track transmission. Muhammad Adil et al. [111] develop the Energy Gauge Node (EGN) strategy to reduce energy consumption by making efficient use of sensor nodes and resolving load-balancing issues. 
Table 2. Load balancing approaches and their limitations

\begin{tabular}{|c|c|c|c|}
\hline Approach & Year & Feature & Limitation \\
\hline Comprehensive Weight Value [89] & 2003 & $\begin{array}{l}\text { Load balancing using } \\
\text { optimization threshold }\end{array}$ & Need to develop balanced clusters \\
\hline Mobile CHs positioning [90] & 2006 & $\begin{array}{l}\text { Balancing traffic load in hybrid } \\
\text { network }\end{array}$ & $\begin{array}{l}\text { Considers the location where the } \\
\text { node is deployed }\end{array}$ \\
\hline $\begin{array}{l}\text { Load balancing with dense clusters } \\
\text { [91] }\end{array}$ & 2007 & $\begin{array}{l}\text { Considers the traffic load of the } \\
\text { entire network }\end{array}$ & $\begin{array}{l}\text { Each node must be aware of the } \\
\text { network }\end{array}$ \\
\hline Load balanced Group Clustering [92] & 2010 & $\begin{array}{l}\text { Implementing dynamic route } \\
\text { calculation based on energy of } \\
\text { the network }\end{array}$ & Considers battery power \\
\hline $\begin{array}{l}\text { Combined TSP-Reduce Algorithm } \\
\text { [93] }\end{array}$ & 2016 & $\begin{array}{l}\text { Find overlapping points of the } \\
\text { nodes }\end{array}$ & High cost of communication \\
\hline Data Dissemination Scheme [94] & 2016 & $\begin{array}{l}\text { Load balancing in UAV } \\
\text { coordinated WSNs }\end{array}$ & Complexity in implementation \\
\hline Light-path provision technique [95] & 2018 & $\begin{array}{l}\text { Load balancing based on } \\
\text { scheduling modulation }\end{array}$ & Based on the traffic of the network \\
\hline $\begin{array}{l}\text { Analytical model for load balancing } \\
\text { [96] }\end{array}$ & 2018 & Optimal path selection & $\begin{array}{l}\text { Needs the energy consumption } \\
\text { details of every node in the } \\
\text { network }\end{array}$ \\
\hline $\begin{array}{l}\text { Multi-path Relay Communication and } \\
\text { Multi-path Weighted Revenue } \\
\text { Algorithm [97] }\end{array}$ & 2018 & $\begin{array}{l}\text { Load balancing based on route } \\
\text { information }\end{array}$ & $\begin{array}{l}\text { All nodes must involve in } \\
\text { establishing routes which } \\
\text { consumes more energy } \\
\end{array}$ \\
\hline Software Define Method [98] & 2018 & $\begin{array}{l}\text { Uses software configuration of } \\
\text { sensors }\end{array}$ & Complex deployment \\
\hline $\begin{array}{l}\text { Enhanced Hybrid Multipath Routing } \\
\text { [99] }\end{array}$ & 2019 & $\begin{array}{l}\text { Inherent clustering and single } \\
\text { load balancing }\end{array}$ & Computational complexity \\
\hline $\begin{array}{l}\text { Distributed Energy-efficient Adaptive } \\
\text { Clustering Protocol [100] }\end{array}$ & 2019 & $\begin{array}{l}\text { Load balancing with self } \\
\text { adaption }\end{array}$ & $\begin{array}{l}\text { Needs to balance energy between } \\
\text { individual nodes in the network }\end{array}$ \\
\hline $\begin{array}{l}\text { Evolutionary Multipath Energy } \\
\text { Efficient Routing Protocol [101] }\end{array}$ & 2019 & $\begin{array}{l}\text { Optimal selection of CHs based } \\
\text { on cuckoo search }\end{array}$ & $\begin{array}{l}\text { Optimization of the network } \\
\text { depends on the power supplied }\end{array}$ \\
\hline Enhanced Clustering Hierarchy [102] & 2019 & Sleep/wake-up mechanism & Needs effective duty cycles \\
\hline $\begin{array}{l}\text { Energy-efficient Load Balancing Ant- } \\
\text { based Routing [103] }\end{array}$ & 2019 & $\begin{array}{l}\text { Uses Ant-based routing } \\
\text { Algorithm }\end{array}$ & $\begin{array}{l}\text { Energy metrics need to be } \\
\text { expected in advance }\end{array}$ \\
\hline $\begin{array}{l}\text { Energy-efficient Load Balancing } \\
\text { [104] }\end{array}$ & 2019 & $\begin{array}{l}\text { Path Distance information of the } \\
\text { nodes is collected }\end{array}$ & More computations involved \\
\hline Hierarchical data job scheduling [105] & 2019 & Prioritizes task scheduling & Should avoid task starvation \\
\hline $\begin{array}{l}\text { Task distribution technique for load } \\
\text { balancing [106] }\end{array}$ & 2020 & Parallel task scheduling & $\begin{array}{l}\text { Depends on the decision making } \\
\text { process of wireless nodes }\end{array}$ \\
\hline $\begin{array}{l}\text { Neighbour Discovery Algorithm } \\
\text { [107] }\end{array}$ & 2020 & $\begin{array}{l}\text { Discovers neighbours using Hop } \\
\text { count }\end{array}$ & $\begin{array}{l}\text { The base station is responsible of } \\
\text { updating the Hop count to the } \\
\text { nodes }\end{array}$ \\
\hline EHMR [108] & 2020 & $\begin{array}{l}\text { Based on static connections in } \\
\text { multi-hop networks }\end{array}$ & $\begin{array}{l}\text { Depends on the density of the } \\
\text { network }\end{array}$ \\
\hline $\begin{array}{l}\text { Load balancing using Breadth First } \\
\text { Search and Dijkstra's Algorithm [109] }\end{array}$ & 2020 & Finding shortest path & $\begin{array}{l}\text { Needs to lower hot spots in the } \\
\text { network }\end{array}$ \\
\hline $\begin{array}{l}\text { Heuristic Load Balancing Algorithm } \\
\text { [110] }\end{array}$ & 2020 & $\begin{array}{l}\text { Non-linear programming based } \\
\text { on network topology }\end{array}$ & Based on transmission power \\
\hline $\begin{array}{l}\text { Load balancing using Energy Gauge } \\
\text { Nodes [111] }\end{array}$ & 2020 & $\begin{array}{l}\text { Energy-balanced joint routing } \\
\text { and Asynchronous service cycles }\end{array}$ & $\begin{array}{l}\text { Depends completely on the } \\
\text { deployment of EGN nodes }\end{array}$ \\
\hline $\begin{array}{l}\text { Lightweight load-balanced and } \\
\text { authentication scheme [112] }\end{array}$ & 2021 & $\begin{array}{l}\text { Based on security authentication } \\
\text { and deployment }\end{array}$ & Control packets overhead \\
\hline
\end{tabular}

EGN nodes are also installed in the WSNs of ordinary nodes because these nodes have a large battery capacity, a high computing power, a reasonable communication speed, and sufficient storage space. The EGN nodes in the network are in charge of disposing of node waste energy and transferring information on the network's Hop set. Path requests initiated by EGN nodes and route-respond packets recognized by ordinary nodes are the two types of packets used for this purpose. To 
calculate the residual energy of the nodes, EGN nodes use the node ID, signal energy, and round trip time set in route response packets. Initially, any typical node in the network assigns maximum energy and is assumed to be efficient, and the energy of the nodes drains after propagation time and falls into a regular or critical category. The sleeping period is tracked by EGN nodes based on residual energy measured by the EGN nodes. Prior to the total number of node failures, the active nodes are extremely efficient. The network is involved in this strategy for an extended time period. Energy-balanced joint routing and asynchronous service cycles are recommended for load handling, which leads to improved network existence.

A lightweight secure load and energy balancing scheme proposed by J il i a n g $\mathrm{Zhou}$ and Lin [112] is used to achieve secure load balancing on the basis of security authentication and deployment. It checks the load and energy for each $\mathrm{CH}$ using four parameters that define the load, computational capacity, and energy for each $\mathrm{CH}$. If a $\mathrm{CH}$ is overloaded, it will broadcast a control packet to the adjacent $\mathrm{CHs}$ containing information about its ID, load, and residual energy. Load and energy balancing request packets are received by each $\mathrm{CH}$ from its neighbours. The adjacent $\mathrm{CH}$ compares the receiving ID to its own database, and if a match is found, it examines the load information and residual energy from the control packets to prevent a network attack. During data transmission between $\mathrm{CHs}$, it employs encryption and decryption techniques. Nagarajan Munusamy and S ri n i v a s a n [113] compare Random Square Grid and Tri-Hexagon Tiling for load deployment in WSN based on coverage, energy consumption, and worst-case delay and concludes that Tri-Hexagon Tiling performs better in terms of load balancing. Table 2 depicts the load balancing approaches in WSN as well as their limitations.

\section{Conclusion}

As we all know, wireless sensor networks face the challenge of balancing energy consumption in order to contribute to the network's longevity. This paper has discussed the challenging issues in wireless sensor networks. We have discussed a few of the parameters that influence energy consumption, such as clustering, routing, media access, and load balancing. We talked about the various existing methodologies for these parameters and how they help with energy conservation. We briefly discussed the benefits, drawbacks, and limitations of these parameters in order to provide an idea of how these existing methodologies might be used in future research. This can also provide inspiration for choosing specific methodologies to improve performance in wireless sensor networks.

\section{References}

1. Ezhilarasi, M., V. Krishnaveni. A Survey on Wireless Sensor Network: Energy and Lifetime Perspective. - Taga Journal, Vol. 14, 2018, pp. 3099-3113. ISSN: 1748-0345.

2. $\mathrm{N}$ a g a r a j a n, M., S. K a r t h i k e y a n. A New Approach to Increase the Lifetime and Efficiency of Wireless Sensor Network. - In: Proc. of IEEE International Conference of Pattern Recognition, Informatics and Medical Engineering (PRIME), 2012, pp. 231-235. 
3. A y a di, H., A. Zou in khi, B. B o u s s a id, M. N. A b d e $1 \mathrm{krim}$, T. V a l. Energy Efficiency in WSN: IEEE 802.15.4. - In: Proc. of 17th International Conference on Sciences and Techniques of Automatic Control and Computer Engineering (STA), Tunisia, 2016, pp. 766-771. DOI:10.1109/STA.2016.7952060.

4. Rault, T., A. M. Bouabdallah, Y. Challah. Energy Efficiency in Wireless Sensor Networks: A Top-Down Survey. - Computer Networks, Vol. 67, 2014, pp. 104-122. https://doi.org/10.1016/j.comnet.2014.03.027

5. A n i t h a, C. S., R. M. S u r e s h. Improving QoS Routing in Hybrid Wireless Mesh Networks Using Cross Layer Interaction and MAC Scheduling. - Cybernetics and Information Technologies, Vol. 15, 2015, No 3, pp. 52-67.

6. S ingh, V. K., R. K u m a r, S. S a h a n a. To Enhance the Reliability and Energy Efficiency of WSN Using New Clustering Approach. - In: Proc. of International Conference on Computing, Communication and Automation (ICCCA'17), IEEE, 2017, pp. 488-493. DOI: 10.1109/CCAA.2017.8229849.

7. W a n g, Y. Distributed Energy Balance Clustering Algorithm in Wireless Sensor Networks. Cybernetics and Information Technologies, Vol. 13, 2013, Special Issue, pp. 88-99.

8. J i a n g, C., D. Y u a n, Y. Z h a o. Towards Clustering Algorithms in Wireless Sensor Networks a Survey. - In: Proc. of IEEE Wireless Communications and Networking Conference, Hungary, 2009, pp. 1-6. DOI: 10.1109/WCNC.2009.4917996.

9. R a thn a, R. Simple Clustering in Wireless Sensor Network. - Cybernetics and Information Technologies, Vol. 16, 2016, No 1, pp. 57-72.

10. L i u, X. A Survey on Clutering Routing Protocols in Wireless Sensor Networks. - Sensors, Vol. 12, 2012, Issue 8, pp.11113-11153. https://doi.org/10.3390/s120811113.

11. F u, C., Z. J i a n g, W. W e i, A. W e i. An Energy Balanced Algorithm of LEACH Protocol in WSN. - International Journal of Computer Science, Vol. 10, 2013, Issue 1, pp. 354-359. ISSN: 1694-0814.

12. Tripath i, M., M. S. G a u r, V. L a x m i, R. B. B a t u 1 a. Energy Efficient Leach-C Prorocol for Wireless Sensor Network. - In: Proc. of 3rd International Conference on Computational Intelligence and Information Technology (CIIT'13), Mumbai, India, 2013, pp. 402-405. DOI: $10.1049 / \mathrm{cp} .2013 .2620$.

13. T a n g, B., D. W a n g, H. Z h a n g. A Centralized Clustering Geographic Energy Aware Routing for Wireless Sensor Networks. - In: Proc. of IEEE International Conference on Systems, Man and Cybernetics, IEEE, Manchester, UK, 2013, pp. 1-6. DOI: 10.1109/SMC.2013.8.

14. C h a n d, S., S. S i n g h, B. K u m a r. Heterogenous HEED Protocol for Wireless Sensor Networks. - Wireless Personal Communications, 2014, No 77, pp. 2117-2139.

15. H a n, Z., J. W u, J. Z h a n g, L. L i u. A General Self Organized Tree Based Energy Balance Routing Protocol for Wireless Sensor Networks. - IEEE Transactions on Nuclear Science, Vol. 61, 2014, Issue 2, pp. 732-740. DOI: 10.1109/TNS.2014.2309351.

16. S h a r m a, I., R. S ing h, M. K h u ran a. Performance Evaluation of PEGASIS Protocol for WSN Using NS2. - In: Proc. of International Conference on Advanced Engineering and Applications, IEEE, Ghaziabad, India, 2015, pp. 926-929. DOI: 10.1109/ICACEA.2015.7164838.

17. Z a r g h a m, A. Deterministic Energy Efficient Clustering Protocol for Wireless Sensor Network Using Evolutionary Optimization Technique. - International Journal of Engineering Development and Research, Vol. 4, 2016, Issue 3, pp. 710-719. ISSN: 2321-9939.

18. B o m g n i, A., B., E. T.F u t e, M. L. S i n d j o u n g, C. T. D j a m e g n i. A Tree-Based Distributed Permutation Routing Protocol in Multi-Hop Wireless Sensor Network. - Wireless Sensor Networks, Vol. 8, 2016, No 6, pp. 93-105.

19. S a b e t, M., H. N a j i. An Energy Efficient Multi-Level Route-Aware Clustering Algorithm for Wireless Sensor Networks: A Self-Organized Approach. - Computers and Electrical Engineering, Vol. 56, 2016, No 1, pp. 399-417.

20. I s t w a 1, Y., S. K. V e r m a. Dual Clustering Head Routing Protocol in WSN. - In: Proc. of 8th International Conference on Computing, Communication and Network Technologies (ICCCNT'17), IEEE, New Delhi, India, 2017, pp. 1-6. DOI: 10.1109/ICCCNT.2017.8203940. 
21. S in g h, S., A. M a 1 i k. Heterogenous SEP Protocol for Increasing Lifetime in WSN. - Journal of Information and Optimization Sciences, Vol. 38, 2017, Issue 5, pp. 721-743.

22. P e n g, K. A Survey of Energy-Efficient Data Gathering in Wireless Sensor Networks. - Journal of Software Engineering, Vol. 11, 2017, Issue 1, pp. 94-101. ISSN:1819-4311.

23. H a n, G., L. Z h a n g. WPO-EECRP: An Energy-Efficient Clustering Routing Protocol Based on Weighting and Parameter Optimization in WSN. - Wireless Personal Communication, Vol. 98, 2017, No 1, pp. 1171-1205.

24. M a z u m d a r, N., H. O m. DUCR: Distributed Unequal Cluster-Based Routing Algorithm for Heterogenous Wireless Sensor Networks. - International Journal of Communication Systems, Vol. 30, 2017, No 18, pp. 1-14.

25. S a r a n y a, V., S. S h a n k a r, G. R. K a n a g a c h i d a m b a r e s a n. Energy Efficient Clustering Scheme (EECS) for Wireless Sensor Networks with Mobile Sink. - Wireless Personal Communications, 2018, No 100, pp. 1553-1567.

26. D a rabkh, K. A., N. F. A l-M a i t a h, I. E. J a f a r, A. F. Kh a lif e h. EA-CRP: A Novel Energy-Aware Clustering and Routing Protocol in Wireless Sensor Networks. - Computers and Electrical Engineering, Vol. 72, 2018, pp. 702-718.

27. Z h o u, W., B. Y u. An Energy Hole Alleviating Algorithm for Wireless Sensor Networks Based on Energy-Balanced Clustering Protocol. - Communications and Computer Information Science, Vol. 2, 2018, No 1, pp. 103-116.

28. Zh ang, Y., M. Li u, Q. Li u. An Energy-Balanced Clustering Protocol Based on Improved CFSFDP Algorithm for Wireless Sensor Networks. - Sensors, Vol. 18, 2018, No 3, pp. 831-896.

29. Hos e n, A., G. Ch o. An Energy Centric Cluster-Based Routing Protocol for Wireless Sensor Networks. - Sensors, Vol. 18, 2018, No 5, pp. 1520-1532.

30. R a m l u c k u n, M., V. B a s s o. An Energy-Efficient Chain-Cluster Based Intelligent Routing Technique for Wireless Sensor Networks. - Applications Computing Information, Vol. 2, 2018, No 1, pp. 78-89.

31. B r i a, R., A. W a h a b, M. A l a y d r u s. Energy Efficient Analysis of TEEN Routing Protocol with Isolated Nodes. - In: Proc. of 4th International Conference on Informatics and Computing (ICIC'19), IEEE, Semarang, Indonesia, 2019, pp. 1-5. DOI: $10.1109 /$ ICIC47613.2019.8985668.

32. D e n e r, M. A New Energy Efficient Hierarchical Routing Protocol for Wireless Sensor Networks. - Wireless Personal Communications, 2018, No 101, pp. 269-286.

33. C h a r a n, P., T. U s m a n i, R. P a u l u s, S. H. S a e e d. Performance of Distributed Energy Aware Routing (DEAR) Protocol with Cooperative Caching for Wireless Sensor Networks. Wireless Sensor Networks, Vol. 11, 2019, No 3, pp. 35-45.

34. D a rabkh, K. A., M. Z. E 1-Y abroudi, A. H. E 1-M ou s a. BPA-CRP: A Balanced Power Aware Clustering and Routing Protocol for Wireless Sensor Networks. - Ad Hoc Networks, Vol. 82, 2019, pp. 155-171.

35. S e n g a th i r, J., P. M. D e v a. An Energy-Proficient Clustering Inspired Routing Protocol Using Improved Bkd-Tree for Enhanced Node Stability and Network Lifetime in Wireless Sensor Networks. - International Journal of Communication Systems, Vol. 33, 2020, Issue 16, pp. 1-17. DOI: $10.1002 /$ dac. 4575 .

36. Mous sa, N., Z.Hamidi-Ala ou i, A.El Belrhti El Ala ou i. ECRP: An Energy Aware Cluster Based Routing Protocol for Wireless Sensor Networks. - Wireless Networks, Vol. 26, 2020, pp. 2915-2928. https://doi.org/10.1007/S11276-019-02247-5

37. S a li m, A., A. A. B a d r a n. Effective Chain-Based Routing Algorithm for Wireless Sensor Networks. - Journal of Computational and Theoritical Nanoscience, Vol. 14, 2017, No 1, pp. 728-735.

38. G a o, Q., D. J. H o 1 d i n g, Y. P e n g, K. J. B 1 o w. Energy Efficiency Design Challenges in Sensor Networks. - In: Proc. of LCS, 2002, pp. 69-72.

39. A m u th a, J., S. S h a r m a, J. N a g a r. WSN Strategies Based on Sensors, Deployment, Sensing Models, Coverage and Energy Efficiency: Review, Approaches and Open Issues. - In: Wireless Personal Communications. Springer, 2019, pp. 1089-1115. https://doi.org/10.1007/s11277-019-06903-z 
40. J u r e n o k s, A., L. N ovi cki s. Analysis of Wireless Sensor Network Structure and Lifetime. Communication and Information Technologies (KIT), Vysoke Tatry, Slovakia, 2017, pp. 1-6. DOI: 10.23919/KIT.2017.8109448.

41. C u i, S., A. G o ld s m i th, A. B a h a i. Energy Constrained Modulation Optimization. - In: IEEE Transactions on Wireless Communications, 2005, No 4, pp. 2349-2360.

42. J u n g, J. W., W. W a n g, M. A. I n g r a m. Cooperative Transmission Range Extension for DutyCycle Limited Wireless Sensor Networks. - In: Proc. of International Conference on Wireless Communication, 2011, pp. 1-5.

43. J a y a w e e ra, S. Virtual MIMO Based Cooperative Communication for Energy Constrained Wireless Sensor Networks. - IEEE Transactions on Wireless Communications, No 5, pp. 984-989.

44. Y u, C. Low Cost Locating Method of Wireless Sensor Network in Precision Agriculture. Cybernetics and Information Technologies, Vol. 16, 2016, No 6, pp. 123-132.

45. X i e, R., A. Li u, J. G a o. A Residual Energy Aware Schedule Scheme for WSNs Employing Adjustable Awake/Sleep Duty Cycle. - Wireless Personal Communications, 2016, No 20, pp. 1859-1887.

46. Y a n g, X., W. Z h a n g. Improved DV-Hop Localization Algorithm Based on Bat Algorithm. Cybernetics and Information Technologies, Vol. 16, 2016, No 1, pp. 89-98.

47. Shakov, V., I. Ko o. Depletion-of-Battery Attack: Specificity, Modelling and Analysis. Sensors, Vol. 18, 2018, Issue 6, p. 1849. https://doi.org/10.3390/s18061849

48. Gn an a pras a mbikai, L., N. Munnus amy. Data Pre-Processing and Classification for Traffic Anomaly Intrusion Detection Using NSLKDD Dataset. - Cybernetics and Information Technologies, Vol. 18, 2018, No 3, pp. 111-119.

49. A f r o z, F., R. B r a u n. Energy-Efficient MAC Protocols for Wireless Sensor Networks: A Survey. - International Journal of Sensor Networks, Vol. 32, 2020, Issue 3, pp. 150-173. DOI: 10.1504/IJSNET.2020.105563.

50. S h a h, I. K., T. M a it y, Y. S. D o h a r e. Algorithm for Energy Consumption Minimization in Wireless Sensor Networks. - IET Communications, Vol. 14, 2019, Issue 8, pp. 1301-1310. ISSN: 1751-8628. DOI: 10.1049/iet-com.2019.0465.

51. S m it a, P., L. S a n th o s h, B. I y e r, P. D e s h p an d e, S. S h a r m a, U. S h i u k a r. An EnergyEfficient MAC Protocol for Wireless Sensor Networks. - In: Advances in Intelligent Systems and Computing. Vol. 1025. Springer, Singapore, 2020, pp. 177-187.

ISBN: 978-981-32-9514-8. https://doi.org/10.1007/978-981-32-95155-17

52. H u a n g, P., L. Li a o, S. S o l t a n i, M. W. Mu kt a, N. Xi. The Evolution of MAC Protocols in Wireless Sensor Networks: A Survey. - IEEE Communication Surveys and Tutorials, Vol. 15, 2013, No 1, pp. 101-120.

53. Qu in tero, V., C. Est e ve z, M. Orch ard, A. P e re z. Improvements of Energy-Efficient Techniques in WSNS: A MAC-Protocol Approach. - IEEE Communication Surveys and Tutorials, Vol. 21, 2018, No 2, pp. 1188-1208. DOI:10.1109/COMST.2018.2875810.

54. Y e, W., J. H e i d e m a n n, D. E s tri n. An Energy-Efficient MAC Protocol for Wireless Sensor Networks. - In: Proc. of INFOCOM 21st Annual Joint Conf. IEEE Computer and Communications Societies Proc. IEEE, New York, NY, USA, 2002, Vol. 3, pp. 1567-1576.

55. S c hurge r s, C., V. T s i t s i s, S. G a n e ri w a l, M. S riv a s t a va. Topology Management of Sensor Networks: Exploiting Latency and Density. - In: Proc. of 3rd Symposium on Mobile Ad Hoc Networking and Computing, ACM. Lausanne, Switzerland, 2002, pp. 135-145.

56. V a n D a m, T., K. L a n g e n d o e n. An Adaptive Energy-Efficient MAC Protocol for Wireless Sensor Networks. - In: Proc. of 1st International Conference on EmbeddedNetworked Sensor Systems, ACM, Los Angeles, California, USA, 2003, pp. 171-180.

57. R a je ndra n, V., K. Obra c zi a, J. J. G a r c i a-L u n a-A c e ve s. Energy-Efficient Collision Free Medium Access Control for Wireless Sensor Networks. - In: Proc. of 1st International Conference on Embedded Networked Sensor Systems (SenSys'03), Los Angeles, California, USA, 2003, pp. 181-192. 
58. L u, G., B. K r i s h n a m a ch a r i, C. S. R a g a v e n d r a. An Adaptive Energy-Efficient and LowLatency MAC for Data Gathering in Wireless Sensor Networks. - In: Proc. of 18th International Parallel and Distributed Processing Symposium, IEEE, Vol. 18, 2004, pp. 3091-3098.

59. L i, J., G. Y. L a z a r o u. A Bit-Map Assisted Energy-Efficient MAC Scheme for Wireless Sensor Networks. - In: Proc. of 3rd International Symposium of IPSN, Berkeley, CA, 2004, pp. 55-60.

60. P o l a stre, J., J. H i 11, D. C u 11 e r. Versatile Power Media for Wireless Sensor Networks. - In: Proc. of 2nd International Conference on Embedded Networked Sensor Systems, ACM, Baltimore, USA, 2004, pp. 95-107.

61. Mille r, M. J., N. H. V a i d y a. Minimizing Energy Consumption in Sensor Networks Using a Wakeup Radio. - In: Proc. of IEEE Wireless Communications and Networking Conference, IEEE, Vol. 4, 2004, pp. 2335-2340.

62. E 1-H o i y di, A., J. D. D e c ot ig n i e. Low Power Downlink MAC Protocols for Infrastructure Wireless Sensor Networks. - Mobile Networks and Applications, Vol. 10, 2005, No 5, pp. 675-690.

63. S u h, C., Y. B. Ko. A Traffic-Aware Energy-Efficient MAC Protocol for Wireless Sensor Networks. - In: Proc. of International Symposium on Circuit Systems (ISCAS'05), Kobe, Japan, 2005, pp. 2975-2978.

64. B u e t t n e r, M., G. V. Y e e, E. A n d e r s o n, R. H a n. X-MAC A Short Preamble Protocol for Duty-Cycled Wireless Sensor Networks University of Colorado at Boulder X-MAC: A Short Preamble Protocol for Duty-Cycled. - Work, Vol. 76, 2006, pp. 307-320.

65. N a m, Y., H. L e e, H. J u n g. An Adaptive MAC Protocol Guaranteeing Network Lifetime for Wireless Sensor Networks Applications. - Computer Communications, Vol. 30, 2007, No 13, pp. 1-7.

66. D u, S., A. K. S a h a, D. B. J o h n s o n. RMAC: A Routing-Enhanced Duty-Cycle MAC Protocol for Wireless Sensor Networks. - In: Proc. of 26th IEEE International Conference on Computer Communications, IEEE INFOCOM, 2007, IEEE, pp. 1478-1486.

67. Sh afiullah, G. M., A. Thompson, P. Wolfs, S. Ali. Energy-Efficient TDMA MAC Protocol for Wireless Sensor Networks Applications. - In: Proc. of 5th ICECE, Bangladesh, 24-27 December 2008, pp. 85-90.

68. K i m, Y., M. S h i n, H. Ch a. Y-MAC: An Energy Efficient Multi-Channel MAC Protocol for Dense Wireless Sensor Networks. - In: Proc. of International Conference on Information Processing in Sensor Networks, IEEE, 2008, pp. 53-63.

69. S u n, Y., O. G u r e w it z, D. B. J o h n s o n. RI-MAC: A Receiver Initiated Asynchronous Duty Cycled MAC Protocol for Dynamic Traffic Loads in Wireless Sensor Networks. - In: Proc. of 6th ACM Conference on Embedded Network Sensor Systems, 2008, ACM, pp. 1-14.

70. Y a d a v, R., S. V a r m a, N. M a 1 a v i y a. Optimized Medium Control Access for Wireless Sensor Network. - International Journal of Computer Science and Network Security, 2008, pp. 334-338.

71. N i a f a r, S., S. S h a h h o s e in i. A New Sink-Based Energy Efficient and Delay Sensitive MAC Protocol for Large Scale WSNs. - In: Proc. of International Symposium on Performance Evaluation Computer Telecommunication Systems (SPECTS'09), 2009, Istanbul, Turkey, Vol. 41, pp. 178-184.

72. Li u, S., K. W. Fan, P. S in ha. CMAC: An Energy Efficient MAC Layer Protocol Using Convergent Packet Forwarding for Wireless Sensor Networks. - ACM Transactions on Sensor Networks, Vol. 5, 2009, No 4, pp. 1-34.

73. Z h e n g, G., J. F u, S. T a n g, Y. L i, Z. D o n g. A Dual Channel Based Energy Efficient and Low Latency MAC Protocol for Wireless Sensor Networks. - In: Proc. of 2nd International Conference on Networks Security, Wireless Communications and Trusted Computing, IEEE, Wuhan, Hubei, China, Vol. 1, 2010, pp. 466-469.

74. T a n g, L., Y. S u n, O. G u r e w it z, D. B. J o h n s o n. PWMAC: An Energy Efficient PredictiveWakeup MAC Protocol 1 for Wireless Sensor Networks. - In: Proc. of IEEE INFOCOM, IEEE, 2011, pp. 1305-1313. 
75. H e n n a, S. SA-RI-MAC: Sender-Assisted Receiver-Initiated Asynchronous Duty Cycle MAC Protocol for Dynamic Traffic Loads in Wireless Sensor Networks. - In: Proc. of International Conference on Mobile Lightweight Wireless Systems, 2011, Springer, Bilbao, Spain, pp. 120-135.

76. M a z 1 o u m, N. S., O. E d f o r s. DCW-MAC: An Energy Efficient Medium Access Scheme Using Duty-Cycled Low Power Wake-Up Receivers. - In: Proc. of IEEE Vehicular Technology Conference (VTC Fall), IEEE, San Francisco, CA, USA, 2011, pp. 1-5.

77. Hefe ida, M. S., T. Canli, A. Khokhar. CL-MAC: A Cross Layer MAC Protocol for Heterogenous Wireless Sensor Networks. - Ad Hoc Networks, Vol. 11, 2013, No 1, pp. 213-225.

78. J a n g, B., J. B. L i m, M. L. S i c h i t i u. An Asynchronous Scheduled MAC Protocol for Wireless Sensor Networks. Computer Networks, Vol. 57, 2013, No 1, pp. 85-98.

79. Ha n, X., L. Shu, Y. Ch e n, H. Z h o u. WX-MAC: An Energy Efficient MAC Protocol for Wireless Sensor Networks. - In: Proc. of IEEE 10th International Conference on Mobile AdHoc and Sensor Systems, 2013, IEEE, Hangzhou, China, pp. 423-424.

80. Li a n g, L., X. L i u, Y. W a n g, W. F e n g, G. Y a n g. SW-MAC: A Low-Latency MAC Protocol with Adaptive Sleeping for Wireless Sensor Networks. - Wireless Personal Communiations, Vol. 77, 2014, No 2, pp. 1191-1211.

81. Morshed, S., G. He ijenk. TR-MAC: An Energy Efficient MAC Protocol Exploiting Transmitted Reference Modulation for Wireless Sensor Networks. - In: Proc. of 17th ACM International Conference on Modeling, Analysis and Simulation of Wireless and Mobile Systems, ACM, 2014, Montreal, QC, Canada, pp. 21-29.

82. L i u, C. J., P. H u a n g, L. X i a o. TAS-MAC: A Traffic Adaptive Synchronous MAC Protocol for Wireless Sensor Networks. - ACM Transactions on Sensor Networks (TOSN), Vol. 12, 2016, No 1, pp. 1-30.

83. Anchora, L., A. Capone, L. Mainetti, V. Mighali, L. Patrono, F. Simone. AS2-MAC: An Energy Efficient MAC Protocol for Wireless Sensor Networks. - Ad Hoc and Sensor Wireless Networks, Vol. 31, 2016, No 1-4, pp. 199-226.

84. U s h a, N. S., M. H a s s e n, S. S a h a. Efficient Duty Cycle Management for Reduction of Energy Consumption in Wireless Sensor Networks. - In: Proc. of International Conference on Electrical and Electronic Engineering (ICEEE'17), 2017, IEEE, pp. 1-4.

85. S u br a m a n i, A. K., I. P a r a m a s i v a m. PRIN: A Priority-Based MAC Protocol for Wireless Sensor Networks Varying the Sample Inter-Arrival Time. - Wireless Personal Communications, Vol. 92, 2017, No 3, Springer, US, pp. 863-881.

86. T o n g, F., J. P a n. Adaptive Data Collection with Free Addressing and Dynamic Duty-Cycling for Sensor Networks. - In: Lecture Notes of the Institute of Computer Sciences, Socio-Informatics and Telecommunications Engineering, Mobile Networks and Applications. Vol. 199. 2017, pp. 212-222

87. Gu $1 \mathrm{sh}$ a n, S., S. K a nd a s a m y. Performance Evaluation of Wireless Sensor Network MAC Protocols with Early Sleep Problem. - International Journal of Communication Networks and Distributed Systems, Vol. 25, 2020, No 2, pp. 123-144.

88. S n e ha, V., M. Nagaraja n. Localization in Wireless Sensor Networks - A Review. Cybernetics and Information Technologies, Vol. 20, 2020, No 4, pp. 3-26.

89. G u p t a, G., M. Y o u n i s. Load-Balanced Clustering of Wireless Sensor Networks. - In: Proc. of IEEE International Conference in Communications, ICC'03, Vol. 3, 2003, pp. 1848-1852.

90. M a, M., Y. Y a n g. Clustering and Load Balancing in Hybrid Sensor Networks with Mobile Cluster Heads. - In: Proc. of 3rd International Conference on Quality of Service in Heterogenous Wired Wireless Networks, ACM, 2006, p. 16.

91. L o w, C. P., C. F a n g, M. N g, Y. H. A n g. Load-Balanced Clustering Algorithms for Wireless Sensor Networks, of Wireless Sensor Networks. - In: Proc. of IEEE International Conference in Communications, 2007, pp. 3485-3490.

92. De n g, Y., Y. Hu. A Load-Balanced Clustering Algorithm for Heterogenous Wireless Sensor Networks. - In: Proc. of IEEE International Conference in e-Product, e-Service and e-Entertainment (ICEEE'10), 2010, pp. 1-4. 
93. Ch en g, C. F., C. F. Y u. Data Gathering in Wireless Sensor Networks: A Combine TSP Reduce Approach. - IEEE Transactions Vehicular Technology, Vol. 65, 2016, No 4, pp. 2309-2324.

94. S harma, V., I. Y o u, R. Ku mar. Energy Efficient Data Dissemination in Multi-UAV Coordinated Wireless Sensor Networks. - Mobile Information Systems, Vol. 2016, 2016, pp. 1-13.

95. Li, Z., J. Z h a n g, Y. L i, L. B a i, Y. J i. Joint Jobs Cheduling and Light Path Provisioning in Fog Computing Micro Data Center Networks. - Journal of Optimized Communication Networks, Vol. 10, 2018, No 7, p. 152.

96. S in g h T o o r, A., A. K. J a i n. Energy Aware Cluster Based Multi-Hop: A New Energy Efficient Routing Protocol for Wireless Sensor Networks. - Sensor Letters, Vol. 16, 2018, No 4, pp. 304-310.

97. M a i o, Y., Z. S u n, N. W a n g, Y. C a o, H. C ru i c k s h a n k. Time Efficient Data Collection with Mobile Sink and MIMO Technique in Wireless Sensor Networks. - IEEE System Journal, Vol. 12, 2018, No 1, pp. 639-647.

98. F a n g, S., Z. C a i, W. S u n, A. L i u, F. L i u, Z. Li a n g, G. W a n g. Feature Selection Method Based on Class Discriminative Degree for Intelligent Medical Diagnosis. - Computer Materials Continua, Vol. 55, 2018, No 3, pp. 419-433.

99. B a la subramanian, D. L., V. Govind as a my. Study on Evolutionary Approaches for Improving the Energy Efficiency of Wireless Sensor Applications. - EAI Endorsed Transactions on Internet of Things, Vol. 5, 2019, Issue 20.

100. Gherbi, C., Z. Aliouat, M. B en mohammed. A Novel Load Balancing Scheduling Algorithm for Wireless Sensor Networks. - Journal of Network Systems Management, Vol. 27, 2019, No 2, pp. 430-462.

101. E z hil ar a si, M., V. Kri s h n a v e n i. An Evolutionary Multipath Energy-Efficient Routing Protocol (EMEER) for Network Lifetime Enhancement in Wireless Sensor Networks. - In: Soft Computing. 2019, pp. 8367-8377.

\section{https://doi.org/10.1007/s00500-019-03928-1}

102. E 1 A l a m i, H., A. N a j i d. ECH: An Enhanced Clustering Hierarchy Approach to Maximize Lifetime of Wireless Sensor Networks. - In: IEEE Access. Vol. 7. 2019, pp. 107142-107153.

103. Li, X., B. K e e g a n, F. M te n z i, T. W e i s e, M. T a n. Energy Efficient Load Balancing Ant Based Routing Algorithm for Wireless Sensor Networks. - In: IEEE Access. Vol. 7. 2019, pp. 113182-113196.

104. B e n A is sa, Y., A. B a chir, M. Khalgui, A. Koubaa, Z. Li, T. Qu. On Feasibility of Multichannel Reconfigurable Wireless Sensor Networksunder Real-Time and Energy Constraints. - IEEE Transactions Systems, Man, Cybernetic Systems, Early Access, 2019. DOI: 10.1109/TSMC.2019.2897166.

105. S u n, Z., L. L i, X. X i n g, Z. L v, N. N. X i o n g. A Novel Node Deployment Assignment Scheme with Data Association Attributed in Wireless Sensor Networks, Journal of Internet Technology, Vol. 20, 2019, No 2, pp. 509-520.

106. W a n g, T., H. L u o, A. J i a, A. L i u, M. X i e. MTES: An Intelligent Trust Evaluation Scheme in Sensor-Cloud-Enabled Industrial Internet of Things. - Trans. Ind. Informat, Vol. 16, 2020, No 3, pp. 2054-2062.

107. N a u shad, A., G. A b b as, S. A. Shah, Z. H. Abbas. Energy Efficient Clustering with Reliable and Load-Balanced Multipath Routing for Wireless Sensor Networks. - In: Proc. of 3rd International Conference on Advancements in Computational Sciences (ICACS'20), IEEE, Pakistan, 2020, pp. 1-9. DOI: 10.1109/ICACS47775.2020.9055957.

108. S a ch a n, S., M. V at s, A. Mish ra, S. Choudh ary. Comparitive Analysis of Clustering Algorithm for WSN. - Intelligent Computing and Engineering, 2020, pp. 63-71.

109. P a n j a, A. K., A. G h o s h. Qualitative Survey on Sensor Node Deployment, Load Balancing and Energy Utilization in Sensor Network. - Nature Inspired Computing for Wireless Sensor Networks, Springer, 2020, pp. 259-277. https://doi.org/10.1007/978-981-15-2125-6_11

110. R a j p o ot, P., P. D wive di. Optimized and Load Balanced Clustering for Wireless Sensor Network to Increase the Lifetime of WSN Using MADM Approaches. - Wireless Networks, 2020, No 26, pp. 215-251.

https://doi.org/10/1007/s11276-018-1812-2 
111. Ad il, M., R. Kh a n, M. A. A $1 \mathrm{~m}$ a i a h, M. B in s aw a d, J. A li, A. A. S a a id a h, Q. Th a h, H. T a. An Efficient Load Balancing Scheme of Energy Guage Nodes to Maximize the Lifespan of Constraint Oriented Networks. - In: IEEE Access. Vol. 8. 2020, pp. 148510-148527. DOI: 10.1109/ACCESS.2020.3015941.

112. Z h o u, J., Z. L i n. Light Weight Load-Balanced and Authentication Scheme for a Cluster Based Wireless Sensor Networks. - International Journal of Distributed Sensor Networks, Vol. 17, 2021, No 2. DOI: $10.1177 / 1550147720980326$.

113. M u n u s a m y, N., K. S r in i v a s a n. Various Node Deployment Strategies in Wireless Sensor Network, IPASJ. - International Journal of Computer Science (IIJCS), Vol. 5, 2017, Issue 8, pp. 039-044.

Received: 30.01.2021; Second Version: 24.03.2021; Third Version: 01.04.2021; Accepted: 10.05.2021 